\title{
Imperative im Deutschen: Konstruktionen, Praktiken oder social action formats?
}

\section{Sprachverwendung zwischen Routine und situierter Adaptation ${ }^{1}$}

Im Zentrum von Susanne Günthners Arbeiten steht das Verhältnis zwischen Grammatik und sprachlichem Handeln in der sozialen Interaktion. Ausgangspunkt war für sie Thomas Luckmanns Einsicht, dass routinisierte sprachliche Muster sedimentierte Lösungen für wiederkehrende kommunikative Aufgaben sind (Luckmann 1986). Diese Erkenntnis ist grundlegend für ihr Verständnis sprachlicher Formen, vor allem von grammatischen Konstruktionen (Günthner 2006, 2010a, 2011) und von kommunikativen Gattungen (Günthner/Knoblauch 1994; Günthner/König 2016; Günthner 2018). Schnelligkeit der Verständigung und Erwartbarkeit des Fortgangs der Interaktion erfordern den Rückgriff auf Formen, die sich bereits früher bei der Herstellung von Intersubjektivität bewährt haben (Feilke 1996). Gleichzeitig aber ist die „Spannung zwischen Emergenz und Sedimentierung, zwischen Reproduktion und Modifikation“ (Günthner 2011: 298) konstitutiv für sprachliche Praxis. Susanne Günthner hat in ihren zahlreichen Untersuchungen zu Konstruktionen der gesprochenen Sprache immer wieder gezeigt, wie Konstruktionen an die jeweils spezifischen, aktuellen interaktiven Kontexte angepasst werden (z. B. bzgl. wenn-Konstruktionen und Pseudo-Clefts: Günthner 2010a). Konstruktionen und Gattungen sind daher nicht fixe Einheiten, die in der Verwendung implementiert werden, sondern „Orientierungsmuster“, die sich flexibel „,im jeweiligen Kontext (meist) in enger Abstimmung mit dem Gegenüber entfalten“ (Günthner/König 2016: 181). Die Anpassung an übergeordnete Gattungskontexte, an vorangehende sequenzielle Kontexte und das leibliche Handeln, Sensitivität gegenüber InteraktionspartnerInnen und ihrem simultanen Handeln und die Zeitlichkeit der Online-Produktion sind für die situierte Adaptation sprachlicher Konstruktionen und damit für ihre Offenheit und die Bildung hybrider Formen verantwortlich (Günthner 2010a; s. a. Auer 2000; Deppermann/ Günthner 2015).

1 Ich danke Hardarik Blühdorn für wertvolle Anregungen und Diskussionen zum Thema dieses Aufsatzes. 
Vor dem Hintergrund dieser Erkenntnisse hat Susanne Günthner in ihren Arbeiten Teile einer interaktionalen Konstruktionsgrammatik ausgearbeitet, die nicht, wie die Konstruktionsgrammatik gemeinhin, Konstruktionen als Einheiten des sprachlichen Wissens (z. B. Goldberg 2003) versteht, sondern „Konstruktionen in der kommunikativen Praxis“" (Günthner 2010a) untersucht. Damit tritt neben den Begriff der „Konstruktion“ ein zweiter zentraler Begriff, nämlich der der „Praxis“, oder, bezogen auf einzelne linguistische Phänomene, der der „Praktiken“. Auch dieser Begriff erscheint in Susanne Günthners Arbeiten (z. B. Günthner 2010b, 2016). Damit verbindet sich die Frage, wie die Gegenstände interaktionslinguistischer Untersuchungen konzeptualisiert werden sollen: Handelt es sich um Konstruktionen oder um Praktiken? Ist beides letzten Endes das Gleiche? Und wie verhalten sich diese Konzepte zu einem weiteren, in der interaktionalen Linguistik häufiger verwendeten Konzept, dem des, social action format'?

In diesem Artikel sollen diese Fragen an einem empirischen Fall diskutiert werden. Dargestellt werden die Ergebnisse einer Untersuchung zu Imperativen in der sozialen Interaktion im Deutschen. Zunächst wird kurz auf die theoretische Bestimmung der drei Begriffe, grammatische Konstruktionen', ,Praktiken“ und ,social action formats' eingegangen. Den Hauptteil des Texts bildet die Analyse unterschiedlicher Verwendungen von Imperativen. Die Ergebnisse der Imperativstudie werden abschließend in Bezug auf die terminologische Diskussion interpretiert.

\section{Theoretische Konzeptualisierungen des Zusammenhangs von grammatischen Formen und sprachlichen Handlungen}

In diesem Abschnitt werden die wesentlichen Bestimmungsstücke von grammatischen Konstruktionen, sprachlichen Praktiken und social action formats diskutiert. Der Fokus der Diskussion liegt darauf, wie mit dem jeweiligen Begriff der Zusammenhang zwischen grammatischer Form und Handlung gefasst wird.

\subsection{Grammatische Konstruktionen}

Da inzwischen zahlreiche Einführungen in die Konstruktionsgrammatik (z.B. Croft 2010; Croft/Cruse 2004; Goldberg 2006; mit Bezug auf mündliche Konstruktionen: Deppermann 2006, 2007, 2011; Günthner 2006; Imo 2007; Fischer 2015) 
und in die cognitive grammar (Langacker 2008) vorliegen, verzichte ich an dieser Stelle auf eine ausführlichere Einführung des Ansatzes. Auch auf die Differenzen zwischen verschiedenen konstruktionsgrammatischen Ansätzen wird hier nur eingegangen, soweit sie für die hier zu führende Diskussion relevant sind. Für unsere Zwecke ist wesentlich, dass die Konstruktionsgrammatik eine symbolische Konzeption von Grammatik vertritt, nach der grammatische Konstruktionen Zeichen sind, die eine Form- mit einer Bedeutungsseite verbinden (Langacker 1987: 2; Croft/Cruse 2004: 257-262). Die Formseite kann oberflächenstrukturell bestimmt oder mehr oder weniger schematisch abstrakt sein (Croft/Cruse 2004: 262-265; Goldberg 2006). Die Bedeutungsseite wird in den meisten Untersuchungen als semantisch verstanden; selten werden darüber hinaus pragmatische Aspekte wie informationsstrukturelle Eigenschaften (Goldberg 1995: 141-151 und 175-179) oder Handlungsfunktionen (Kay/Fillmore 1999) mitbetrachtet. Zur Art und Enge der Verknüpfung zwischen Form- und Bedeutungsseite bestehen unterschiedliche Auffassungen, die von Prototypenstrukturiertheit und Polysemie (Goldberg 1995, 2006) über Unterspezifikation (Deppermann/Elstermann 2008) bis hin zu einer konstruktivistischen Sicht reichen, welche die Granularität von Konstruktionsbeschreibungen und folglich ihre Menge und Spezifität von der Perspektive des Analytikers abhängig macht (Imo 2011).

Aus interaktionslinguistischer Sicht wurde immer wieder festgestellt, dass sequenzielle, leibliche, prosodische, interaktionstypologische, gattungsspezifische, stilistische und rhetorische Aspekte in konstruktionsgrammatischen Arbeiten meistens fehlen, nicht zuletzt deshalb, weil sie oft keine Daten von authentischen Vorkommen von Konstruktionen (in der mündlichen Interaktion oder in Texten) untersuchen (Deppermann 2006, 2011; Günthner 2006, 2010a). Deppermann (2011) zieht daraus die Konsequenz, dass die Konstruktionsgrammatik durch andere interaktionslinguistische Methoden und Beschreibungsinstrumente ergänzt werden muss, um zu einer vollständigeren Analyse der Verwendung von Grammatik-in-Interaktion zu kommen. Andere sind dagegen der Auffassung, dass die Konstruktionsgrammatik in der Lage ist, auch all die o. g. Aspekte von Sprachverwendung zu integrieren (z. B. Fischer 2010, 2015; Östman 2015), da es sich letzten Endes sämtlich um Aspekte handele, die als Form-Funktions-Zusammenhänge zu modellieren seien, und da alles sprachverwendungsrelevante Wissen als konstruktionales Wissen zu beschreiben sei. Eine mittlere Position nimmt bspw. Imo (2015) ein. 


\subsection{Sprachliche Praktiken}

Während die Konstruktionsgrammatik ein genuin linguistischer Ansatz ist und ihr Ausgangsproblem die Analyse sprachlicher Formen ist, stammt die Praxistheorie aus philosophisch-soziologischen Theoriekontexten und widmet sich dem leiblichen Handlungsvollzug (vgl. z. B. Bourdieu 1974; Schatzki et al. 2001; Reckwitz 2003). Praktiken teilen mit Konstruktionen, dass sie formbestimmt sind und Routinecharakter haben. Doch spielen für Praktiken eine ganze Reihe weiterer Bestimmungsstücke eine Rolle, die in konstruktionsgrammatischen Analysen selten oder nie in den Blick kommen: die Leiblichkeit und Medialität der Realisierung, soziale Beteiligungsstrukturen, ihr Handlungsbezug, ihre (soziale und situative) Indexikalität und Flexibilität, ihr Kontextbezug und die Temporalität ihres Vollzugs (Deppermann et al. 2016b: 3-10). Wie Konstruktionen werden Praktiken auf sehr unterschiedlichen Granularitätsniveaus angesiedelt (s. Deppermann et al. 2016a). Für die Analyse grammatischer Phänomene bietet sich das konversationsanalytische Verständnis von Praktiken an (Schegloff 1997; Deppermann 1999: 79-84; Heritage 2010), das Praktiken als einen Zusammenhang von Kontexten, Ressourcen und Handlungen sieht: „Praktiken (...) sind kontextsensitiv, als routinisierte Verwendungsweisen von Ressourcen für situierte Handlungen, die flexibel an die je spezifischen Gegebenheiten angepasst werden“ (Selting 2016: 29). Die sequenzielle Position, Beteiligungsstrukturen und häufig auch die Einbettung in größere Handlungszusammenhänge sind dabei konstitutiv für den Vollzug von Praktiken. In den Praktikenbegriff gehen also wesentliche Aspekte ein, deren Abwesenheit in konstruktionsgrammatischen Untersuchungen von Günthner $(2006,2010 a)$ und Deppermann $(2006,2011)$ problematisiert werden. Andererseits weisen die vorliegenden interaktionslinguistischen und konversationsanalytischen Analysen oft nicht die Systematik der linguistischen Beschreibung auf, die konstruktionsgrammatische Arbeiten auszeichnet. Dies liegt daran, dass für das interaktionslinguistische und konversationsanalytische Interesse eine formal bestimmte Handlungspraktik (action formation) im Vordergrund steht, nicht aber die Variation von strukturell ähnlichen Formen und ihre netzwerkartigen Relationen.

\subsection{Social action formats}

Die sprachlichen Formate, die zur Konstruktion bestimmter sprachlicher Handlungen in bestimmten Sequenzkontexten der Interaktion benutzt werden, werden von Fox (2007: 305) als social action formats bezeichnet. Der Begriff social action format unterstreicht, dass bestimmte grammatische Formate routinemäßig zur 
Konstruktion bestimmter Handlungen (action formation) wie Bitten, Vorschläge, Aufforderungen etc. gebraucht werden (Couper-Kuhlen 2014; Couper-Kuhlen/Thompson i.d.B.). Dabei bezieht sich Fox (2007: 304) auf Schegloffs Ansatz der positionally sensitive grammar (Schegloff 1996), nach dem je nach Turnund Sequenzkontext eine andere „micro-syntax“ (Fox/Jaspersen 1995: 114) zur Handlungskonstruktion verwendet wird (s. z. B. Curl 2006 für Angebote im Englischen). Beispiele sind etwa die grammatischen Ressourcen, die zur Konstruktion von Antworten (Raymond 2003) oder für Reaktionen auf Informationen, Bewertungen und Bitten (Thompson et al. 2015) benutzt werden. Handlungen können durch verschiedene social action formats realisiert werden. Die Variation der Formate reflektiert spezifische Bedingungen des Sequenzkontexts, der leiblich-räumlichen Konstellation der Beteiligten oder Ausprägung von in der Situation relevanten Merkmalen der Beteiligten. Unterschiedliche sprachliche Formate der Aufforderung (z. B. modale Deklarative mit want/need, selbst- vs. adressaten-orientierte Interrogative (can you vs. can I), Imperative) indizieren z. B. unterschiedliche Grade des Anspruchs der Berechtigung, des Bezugs auf ein einseitiges Interesse vs. auf ein joint project, des Aufwands, der mit der Erfüllung der Aufforderung verbunden ist, usw. (Fox/Heinemann 2016; Rossi 2015; Zinken 2016). Fox/Heinemann (2016: 3-4) stellen fest, dass social action formats nicht einfach kategoriale Handlungstypen implementieren: „even relatively minor grammatical variations are of systematic relevance for how participants in interaction accomplish the action of requesting.“

\section{Sprachliches Handeln mit Imperativen}

Es ist eine Binsenweisheit, dass Imperative für Aufforderungshandlungen benutzt werden. Nicht ohne Grund werden Imperativsätze auch als „Aufforderungssätze“ bezeichnet (Duden 2016: 903). Allerdings ist dies eine problematische Vermischung von syntaktisch bzw. morphologisch bestimmten Satztypen und Handlungsfunktionen. Denn Aufforderungen können ebenso gut mit Deklarativsätzen (du musst jetzt rechts abfahren), Interrogativsätzen (kannst du jetzt rechts abfahren) oder verschiedenen nicht-sentenziellen Formaten (jetzt rechts rausfahren, jetzt rechts raus, jetzt, los) realisiert werden. Die Frage ist also, worin die Spezifik der Aufforderungshandlungen besteht, die mit Imperativen vollzogen werden.

Die Höflichkeitsforschung geht davon aus, dass Imperative (im Vergleich bspw. zu Interrogativen) unhöflich sind. Nach diesem Ansatz sind es besondere soziale Konstellationen von Aufforderndem und Aufgefordertem, die den Gebrauch von Imperativen legitimieren: ein großes Machtgefälle zwischen Auf- 
forderndem und Aufgefordertem (power-Variable) oder ein besonders enges persönliches Verhältnis (distance-Variable; vgl. Brown/Levinson 1987; Blum-Kulka et al. 1989). Konversationsanalytische Untersuchungen bestätigen diese Sicht teilweise. Craven/Potter (2010) sehen die Berechtigung (entitlement) des Auffordernden, Antaki/Kent (2012) und Mondada (2013) das Wissensgefälle zwischen Aufforderndem und Aufgefordertem als Grundlage für den Gebrauch von Imperativen.

Neuere Untersuchungen zeigen aber, dass die Kategorisierung von Imperativen als "unhöflich" in vielen Fällen unpassend ist und dass ihre Verwendung oft nicht durch die sozialen Merkmale der Beteiligten und ihre Beziehung zueinander motiviert ist. Letzteres wird allein schon dann deutlich, wenn in der gleichen Beteiligtenkonstellation unterschiedliche sprachliche Formate für Aufforderungen benutzt werden. Ein wesentlicher Faktor für die Verwendung von Imperativen sind Zeitparameter des Handelns. Vine (2009) wies darauf hin, dass Imperative insbesondere dann benutzt werden, wenn die Aufforderung sofort umgesetzt werden soll (now-imperatives). Mondada (2011, 2013, 2017, 2018) konnte zeigen, dass Imperative die Dringlichkeit der Reaktion signalisieren, da sonst unerwünschte Konsequenzen zu befürchten sind (z. B. beim gemeinsamen Videospielen, beim Rennfahrertraining, im OP-Saal). Antaki/Kent (2012) und Deppermann $(2015,2018 a)$ zeigen, dass Imperative zur Erneuerung einer Aufforderung benutzt werden, wenn eine zuvor schon gegebene Anweisung nicht (korrekt) ausgeführt wurde oder der Adressat nicht von sich aus das erforderliche und von ihm erwartete Handeln gezeigt hat (zu Letzterem s. a. Stevanovic 2017). Imperative treten daher oft in längeren Sequenzen des Insistierens auf (s. a. Goodwin 2006; Goodwin/Cekaite 2013).

Ein anderer Parameter betrifft die gemeinsame Verantwortlichkeit für ein joint project. Studien zum Deutschen, Italienischen und Polnischen (Rossi 2012, 2015; Zinken/Ogierman 2013; Zinken 2016; Zinken/Deppermann 2017) haben gezeigt, dass Imperative benutzt werden, wenn eine Handlung beauftragt wird, die im Interesse beider Interaktionsteilnehmer liegt bzw. die im Zuständigkeitsbereich des Aufgeforderten (hier zum Finnischen: Rauniomaa 2017) liegt. Dagegen werden andere Formate wie Interrogative benutzt, wenn nur der Auffordernde von der Erfüllung der Aufforderung profitiert (Couper-Kuhlen 2014). Allerdings scheinen hier sprachspezifisch teilweise unterschiedliche Verwendungsgepflogenheiten zu bestehen (s. Zinken/Ogierman 2013 zu Englisch vs. Polnisch). Außerdem spielen die Kombination mit Partikeln (Dänisch: Heinemann/Steensig 2017; Deutsch: Zinken/Deppermann 2017, Finnisch: Sorjonen 2017), Suffixen (Finnisch: Stevanovic 2017) sowie der Verbaspekt (Polnisch: Zinken 2016) eine wichtige Rolle für die genauere Bestimmung der Handlung, die mit dem Imperativ-Turn vollzogen wird. 
Schließlich reflektiert der Gebrauch von Imperativen, dass der Sprecher davon ausgeht, dass der Adressat nicht nur generell im Rahmen eines joint project kooperiert (s. Rossi 2015), sondern auch schon auf die Handlung, zu der aufgefordert wird, (leiblich, räumlich) vororientiert ist (Zinken/Deppermann 2017), diese problemlos ausführen kann und bereit dazu ist (Bolden 2017). Im Kontext der Untersuchung von Instruktionen in der Fahrschule konnte gezeigt werden, dass mit Imperativen zu einfachen, bekannten und im Kontext hochgradig erwartbaren Routinehandlungen aufgefordert wird, während Deklarative (auch) dazu benutzt werden, neues Wissen über auszuführende Handlungen zu vermitteln. Entsprechend ist die sprachliche Gestaltung, insbesondere die Argumentstruktur von deklarativen Instruktionen viel komplexer als die von imperativen (Deppermann 2018a).

\section{Imperative in der verbalen Interaktion im Deutschen}

Im Folgenden berichte ich über die Ergebnisse einer Studie zur Verwendung von Imperativen im Deutschen in verschiedenen Sequenz- und Handlungskontexten. ${ }^{2}$ Die Untersuchung zeigt, dass Imperative zwar in allen Fällen für ein deontisches Handeln benutzt werden, d. h. anzeigen, dass es erwünscht ist, dass eine bestimmte Handlung vollzogen wird. Die genauere Handlungsqualität erweist sich aber als abhängig von kontextuellen Bedingungen und von der sprachlichen Realisierung der Imperativäußerung, insbesondere ihrer Argumentstrukturrealisierung, der Lexikalisierung und dem (Nicht-)Vorkommen der Modalpartikel mal.

Grundlage der Untersuchung ist das Korpus FOLK (Schmidt 2018) in der Version 2.10 (2018) mit 229h Umfang und 2.2 Mio. Wörtern. Mithilfe der Suche nach den Part-of-Speech Tags VVIMP und VAIMP wurden 5967 Imperativformen gefunden. ${ }^{3}$ Tabelle 1 zeigt die häufigsten Lemmata, die in Imperativform vorkamen.

Die Imperativformen komm, guck (mal), hör mal und pass auf werden allerdings sehr häufig als Diskursmarker (DM) benutzt (s. Proske 2014, 2017; Günthner 2017). Diese Fälle sind in der Tabelle mitenthalten, so dass die Zahlen hier deutlich überschätzt sind. Bei den einzelnen Vorkommen ist nicht immer die Trennlinie

2 Zur Morphologie und Bildung von Imperativen im Deutschen siehe Donhauser (1986), Zifonun et al. (1997: 1724-1731) und Raffelsiefen (2002).

3 Die Treffer wurden manuell um falsche Positive bereinigt, falsche Negative wurden hinzugefügt. 
Tab. 1: Häufigste Imperativformen in FOLK

\begin{tabular}{lc}
\hline Lemma & Imperativformen \\
\hline gucken & 907 \\
kommen & 634 \\
machen & 485 \\
warten & 559 \\
lassen & 374 \\
sagen & 289 \\
nehmen & 259 \\
geben & 236 \\
(auf)hören & 194 \\
schauen & 189 \\
gehen & 172 \\
aufpassen & 141 \\
bleiben & 108 \\
stoppen & 104 \\
stellen & 70 \\
rufen & 68 \\
zeigen & 66 \\
schreiben & 64 \\
tun & 59 \\
\hline
\end{tabular}

zwischen Imperativ und Diskursmarker klar zu ziehen. Auch warte wird in Bedeutungen verwendet, die sich von der üblichen Semantik des Verbs unterscheiden (z. B. Suspendieren oder Unterlassen einer Aktivität), aber doch meist näher am Imperativ als am Diskursmarker liegen (Proske 2017).

Tabelle 2 zeigt, dass sich das Vorkommen von Imperativen je nach Interaktionstyp sehr unterscheidet. Am häufigsten finden wir sie in Interaktionen, in denen praktische Handlungen instruiert werden. In Rettungsübungen gibt der Einsatzleiter Instruktionen ${ }^{4}$ und bittet (ebenso wie die Assistenten) um Objekte oder praktische Handlungen. In der Fahrschule fordert der Fahrlehrer zu Fahrhandlungen auf und gibt Anweisungen zur Bedienung des Fahrzeugs (s. De Stefani/Gazin 2014). In Spielinteraktionen wird zu bestimmten Spielhandlungen oder deren Unterlassung aufgefordert. Die Häufigkeit von Imperativen in informellen Alltagsinteraktionen kommt der im Gesamtkorpus am nächsten, auch die Rangreihe der

\footnotetext{
4 Der Begriff, Instruktion“ wird hier spezifisch für Anweisungen, die umgehend zu befolgen sind, verwendet (vgl. Lindwall/Lymer/Greiffenhagen $2015 \mathrm{zu}$ Instruktionsbegriffen). Instruktionen in diesem Sinne sind eine Unterklasse von Aufforderungen. Sie können pädagogisch, im Rahmen der Vermittlung von Wissen und Fertigkeiten oder aufgrund hierarchisch legitimierter Weisungsverhältnisse („deontischer Autorität“, Stevanovic/Peräkylä 2012) realisiert werden.
} 
Tab. 2: Vorkommen von Imperativen in verschiedenen Interaktionstypen

\begin{tabular}{|c|c|c|}
\hline Interaktionstyp & Relative Häufigkeit (IMP/Minuten) & Häufigste Lemmata \\
\hline FOLK gesamt & $0.35(5964 / 16774)$ & s. Tabelle 1 \\
\hline Rettungsübungen & $1.20(115 / 96)$ & $\begin{array}{l}18 \text { machen, } 16 \text { warten, } 13 \text { geben, } \\
11 \text { holen, } 8 \text { gucken, } 8 \text { nehmen, } \\
6 \text { kommen }\end{array}$ \\
\hline $\begin{array}{l}\text { Praktische } \\
\text { Fahrschulstunden }\end{array}$ & $1.18(456 / 383)$ & $\begin{array}{l}134 \text { gucken, } 98 \text { lassen, } \\
40 \text { aufpassen, } 44 \text { (Gas) geben, } \\
35 \text { bleiben, } 35 \text { stoppen }\end{array}$ \\
\hline $\begin{array}{l}\text { Spielinter- } \\
\text { aktionen }\end{array}$ & $0.70(872 / 1253)$ & $\begin{array}{l}127 \text { kommen (meist DM), } \\
106 \text { nehmen, } 103 \text { warten, } \\
71 \text { machen, } 66 \text { gucken, } 52 \text { lassen, } \\
48 \text { sagen, } 47 \text { geben, } 23 \text { (auf)hören, } \\
23 \text { schauen, } 22 \text { gehen, } \\
14 \text { aufpassen, } 14 \text { ziehen }\end{array}$ \\
\hline $\begin{array}{l}\text { Informelle } \\
\text { face-to-face } \\
\text { Alltagsinteraktion }\end{array}$ & $0.47(952 / 2032)$ & $\begin{array}{l}228 \text { gucken (meist DM), } 92 \text { kommen } \\
\text { (meist DM), } 91 \text { (auf)hören, } \\
73 \text { machen, } 56 \text { warten, } 44 \text { sagen, } \\
35 \text { geben, } 35 \text { lassen, } 28 \text { gehen, } \\
24 \text { nehmen, } 17 \text { aufpassen }\end{array}$ \\
\hline $\begin{array}{l}\text { Unterrichtsinter- } \\
\text { aktion }\end{array}$ & $0.20(170 / 871)$ & $\begin{array}{l}24 \text { lesen, } 21 \text { schauen, } 12 \text { machen, } \\
11 \text { lassen, } 11 \text { gucken, } 11 \text { sagen, } \\
10 \text { versuchen }\end{array}$ \\
\hline $\begin{array}{l}\text { Coachinginter- } \\
\text { aktionen }\end{array}$ & $0.11(20 / 185)$ & 4 nennen \\
\hline $\begin{array}{l}\text { Öffentliche } \\
\text { Schlichtung }\end{array}$ & $0.03(37 / 1291)$ & $\begin{array}{l}5 \text { halten, } 4 \text { kommen (alle DM), } \\
3 \text { sagen, } 3 \text { bleiben }\end{array}$ \\
\hline
\end{tabular}

häufigsten Lemmata ähnelt hier der im Gesamtkorpus. Im Schulunterricht kommen Imperative erstaunlicherweise recht selten vor. Hier wird natürlich sehr häufig instruiert, aber es werden vorrangig andere Formate wie Interrogative, Deklarative und Adhortative benutzt. Im Coaching und in öffentlichen Schlichtungsgesprächen sind Imperative sehr selten. In letzteren werden sie fast ausschließlich vom Schlichter zur Gesprächsorganisation benutzt.

Im Folgenden betrachten wir die Verwendung von Imperativen im Kontext der sequenziellen Bearbeitung von joint projects in der sozialen Interaktion. Datengrundlage sind 226 Vorkommen von Imperativen in Videoaufnahmen des Korpus FOLK aus folgenden Interaktionstypen: informelle Alltagsinteraktion, Rettungsübungen, praktische Fahrschulstunden sowie Unterrichtsinteraktionen. Alle Vorkommen wurden detailliert sequenzanalytisch und syntaktisch untersucht. Dabei 
zeigte sich, dass Imperative üblicherweise im Kontext eines bereits begonnenen joint project benutzt werden ( $\mathrm{n}=205 / 226)$. Vier Fälle des Gebrauchs von Imperativen ließen sich hier unterscheiden:

- Es wird ein nächster Schritt des gemeinsamen Handelns auf ein Ziel hin initiiert (4.1);

- es wird auf einer vorangegangenen Aufforderung insistiert (4.2);

- das laufende Handeln das Partners wird korrigiert (4.3);

- eine Initiative des Partners wird ratifiziert bzw. eine Erlaubnis wird erteilt (4.4).

Nur selten fanden sich Fälle, in denen mit einem Imperativ ein neues joint project eröffnet wurde ( $n=21 / 226$; Abschn. 4.5). Dass hier Imperative in einer abweichenden Weise verwendet werden, manifestiert sich in charakteristischen Unterschieden ihrer Turnkonstruktion und syntaktischen Struktur.

\subsection{Initiierung eines nächsten Schritts eines joint project}

Joint projects werden in den untersuchten Daten zumeist mit Deklarativen, Interrogativen oder Adhortativen initiiert, mit denen der neue Handlungskomplex angekündigt wird oder zu einer zu ihm gehörenden Handlung aufgefordert wird. Imperative werden dann benutzt, um einen nächsten Schritt des bereits laufenden joint project zu initiieren. Dabei beziehen sie sich fast immer auf einzelne, einfache Handlungen, kaum einmal auf komplizierte oder zeitlich erstreckte Handlungsabläufe.

Ausschnitt \#1 stammt aus einer Rettungsübung. Der Assistent AS1 will den Kopf des Patienten, der auf einer Treppe liegt, abstützen. In einem Konjunktiv IIKonditional äußert er eine (bisher nicht erfüllte) Bedingung dafür (01-06), was als Bitte um Hilfe verstanden werden kann (Bild 1):

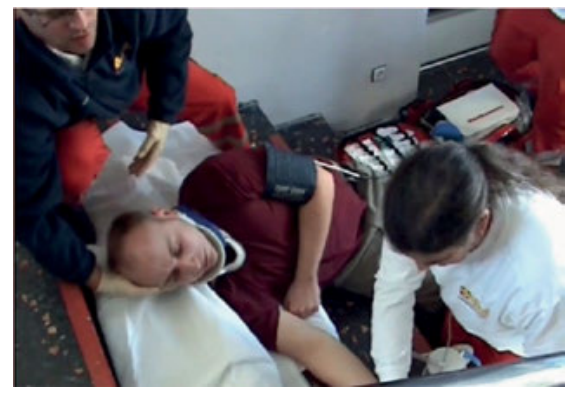

Bild 1: AS1 (links) hält Kopf des Patienten und bittet um Unterlage. 


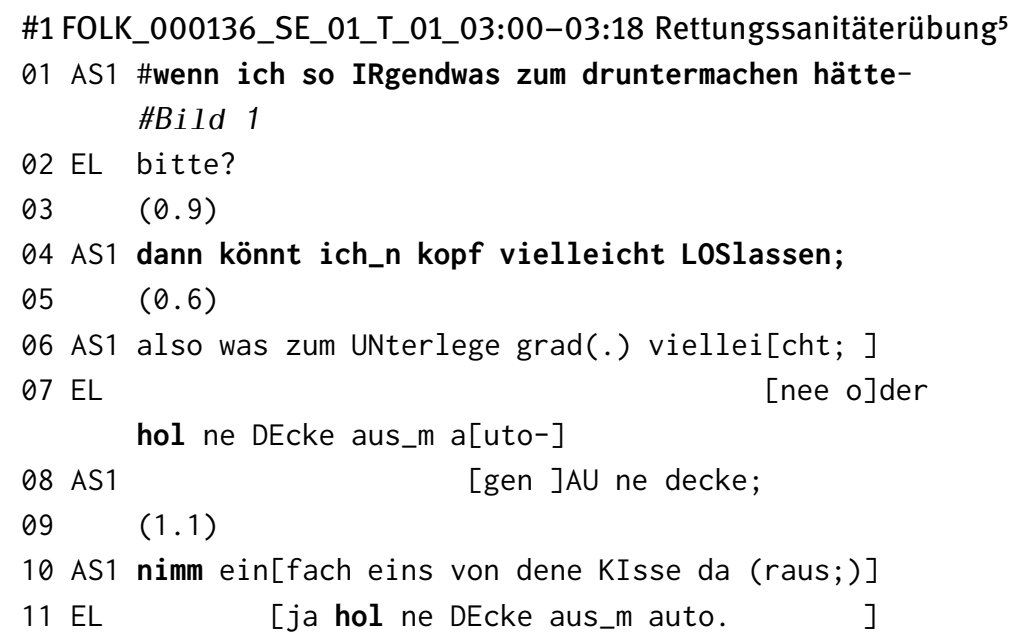

Der komplexe Konditionalsatz in 01-04 eröffnet ein neues joint project, die Abstützung des Kopfs des Patienten. Der Einsatzleiter EL fordert daraufhin den zweiten Assistenten AS2 per Imperativ auf, eine Decke aus dem Rettungswagen zu holen (07). AS1 (in 10) und EL (in 11) formulieren weitere Varianten dieser Aufforderung an AS2 in Imperativen.

In \#2, ebenfalls aus einer Rettungsübung, wird ein neues joint project, das Anbringen einer Ringerlösung an einem Patienten durch eine Frage im Interrogativformat „aber (.) wo_s jetzt die RINger-“ eröffnet.

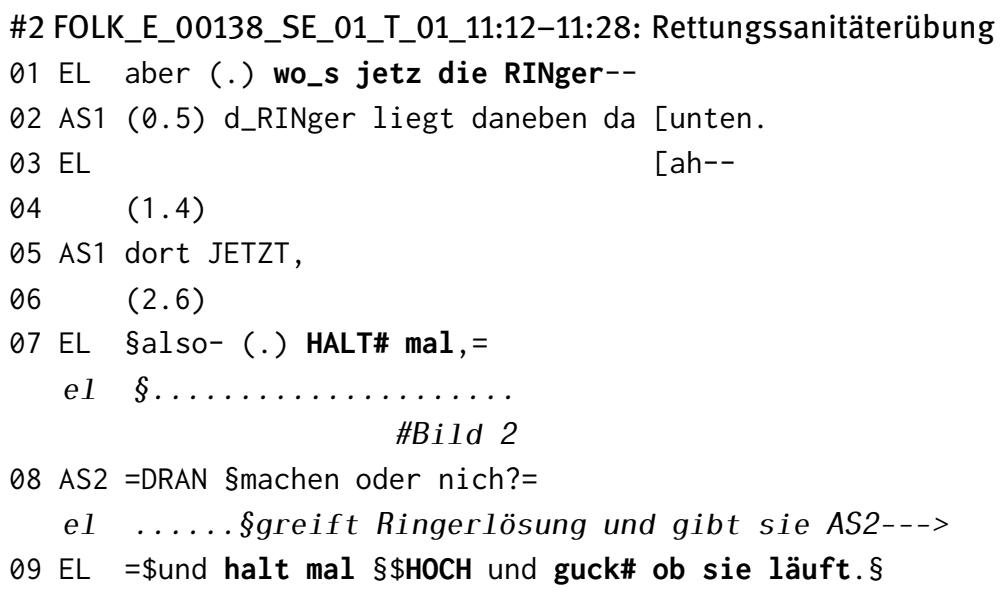

5 In den Transkripten werden jeweils die Turns, mit denen ein joint project eröffnet wird, sowie die Imperativsätze fett gesetzt. 


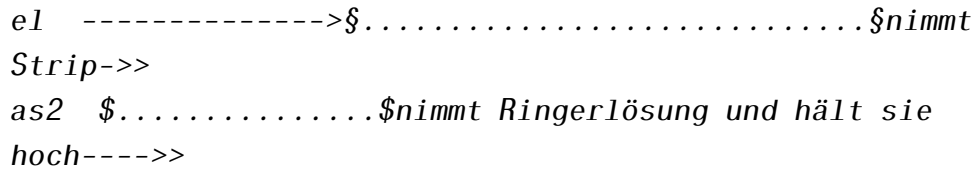

\#Bild 3

Nachdem die Ringerlösung aufgefunden wurde, greift EL nach ihr und gibt sie AS2 (07-09). Seine Aufforderung, die Flasche mit der Lösung zu halten, formuliert er in 07 mit dem Imperativ „halt mal-“. Während der Imperativ anzeigt, dass es um eine Handlung geht, die AS2 problemlos ausführen kann, und dass AS2 auch grundsätzlich für das joint project verfügbar ist, signalisiert die Modalpartikel, dass EL registriert, dass AS2 aktuell noch nicht auf das joint project orientiert ist - AS2 schaut nicht in Richtung der Flasche (Bild 2). Auch die zweite Aufforderung „halt mal HOCH“ (09) dokumentiert mit dem Imperativ, dass AS2 zwar für die anstehende Aufgabe verfügbar ist, mal zeigt aber erneut an, dass er nicht auf die anstehende Aufforderung orientiert ist, denn AS2 hatte eine andere Handlungserwartung artikuliert: „DRAN machen oder nich?“ (08). Als AS2 schließlich die Flasche genommen hat, äußert EL eine dritte imperativische Aufforderung („guck ob sie läuft.“, 09), diesmal ohne Modalisierung, da AS2 die Flasche nun hält und anschaut (Bild 3).

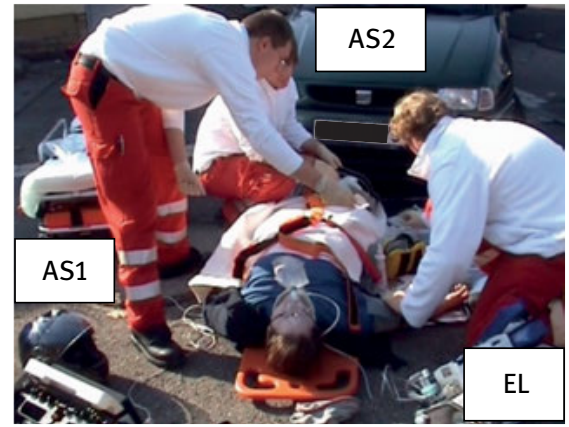

Bild 2: EL bittet AS2 „also halt mal“, während AS2 auf Patienten schaut.

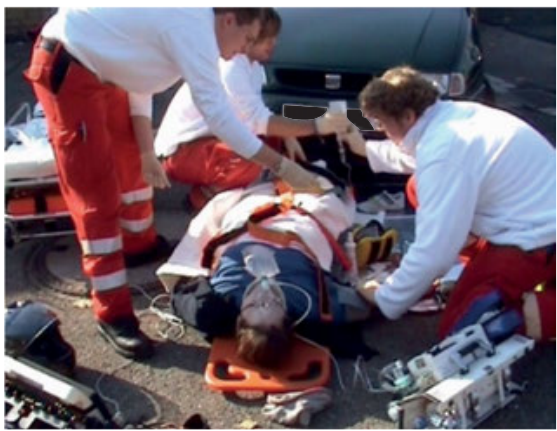

Bild 3: EL bittet AS2 „guck ob sie läuft“, während er ihm die Ringerlösung reicht.

Die Imperative in 07 und 09 („halt mal (HOCH)“) werden jeweils ohne direktes Objekt realisiert. Die reduzierte Argumentstruktur wird durch die strukturelle Latenz (Auer 2014, 2015) des weiterhin salienten Objekts „RINger“ (01) ermöglicht. Diese Salienz ist aber nicht einfach syntaktisch basiert; sie beruht mindestens ebenso sehr auf der Tatsache, dass die Flasche mit der Ringerlösung der fokale Gegen- 
stand des in 01 initiierten joint project ist und für die Beteiligten auch visuell verfügbar ist.

Von den 226 untersuchten Imperativen werden ca. die Hälfte ( $\mathrm{n}=110)$ mit mal produziert. In der Literatur finden wir üblicherweise die Aussage, dass durch mal die mit der Aufforderung verbundene Zumutung abgemildert werde (neben vielen anderen: Bublitz 2003). Dies wird etymologisch durch die Herkunft von mal< ein Mal begründet. In den Daten findet sich für diese Erklärung keine Evidenz abgesehen davon, dass sie unschlüssig erscheint, denn auch ohne mal wird nur zur einmaligen Ausführung der Handlung aufgefordert und die Befolgung der Aufforderung wird durch mal auch nicht weniger verbindlich. Es ist aber festzustellen, dass [Imperativ + mal] immer dann verwendet wird, wenn der Adressat zwar für die Ausführung der beauftragten Handlung verfügbar ist und zumeist auch schon generell das joint project, zu dem die aufgeforderte Handlung beitragen soll, ratifiziert hat, jedoch im Moment der Aufforderung körperlich nicht auf die anstehende Aufgabe orientiert ist. Die fehlende Orientierung auf die anstehende Aufgabe kann darin bestehen, dass er körperlich vom Sprecher oder vom Objekt, das Gegenstand der Aufforderung ist, abgewandt ist, selbst gerade mit etwas anderem beschäftigt ist oder zuvor eine abweichende Erwartung hinsichtlich des nächsten Handlungsschritts zum Ausdruck gebracht hat (Zinken/Deppermann 2017). Aus Sicht des Auffordernden drückt mal also aus, dass er eine unproblematische und legitime, aber im Moment vom Adressaten nicht erwartete Aufforderung formuliert.

Ausschnitt \#3 zeigt einen weiteren Fall, in dem ein joint project (erfolglos) bereits initiiert wurde und ein Imperativ benutzt wird, um seine Realisierung voranzutreiben. Es handelt sich um eine Deutschstunde in einem Wirtschaftsgymnasium, in der Gedichte interpretiert werden sollen. Auf die Eingangsfrage des Lehrers (01/03), mit der er um Meldungen bittet, zeigen die SchülerInnen durch Blickabwendung an, dass sie nicht für die Aufgabe zur Verfügung stehen (s. die Analyse in Deppermann 2018b, Bild 4).

\#3 FOLK124_10:43-10:50 Deutschunterricht im Wirtschaftsgymnasium

01 LE wer traut sich_s zu ne INhaltsangabe zu \#machen?

\#Bild 4

$02 \quad(1.2)$

$03 \mathrm{LE}$ von diesem geDICHT,

$04 \quad(3.0)$

05 LE SCHWIErig, =ne?

$06 \quad(0.4)$

07 LE hm_hm ((schnalzt)) MACHT nichts;

$08{ }^{\circ} \mathrm{h}$ okay ${ }^{\circ} \mathrm{h}$ probier ma_s mal zuSAmmen; 
10 (1.2)

11 LE lina FANG\# mal an, (.)

\#Bild 4

12 LE LIES mal die erste strophe vor,

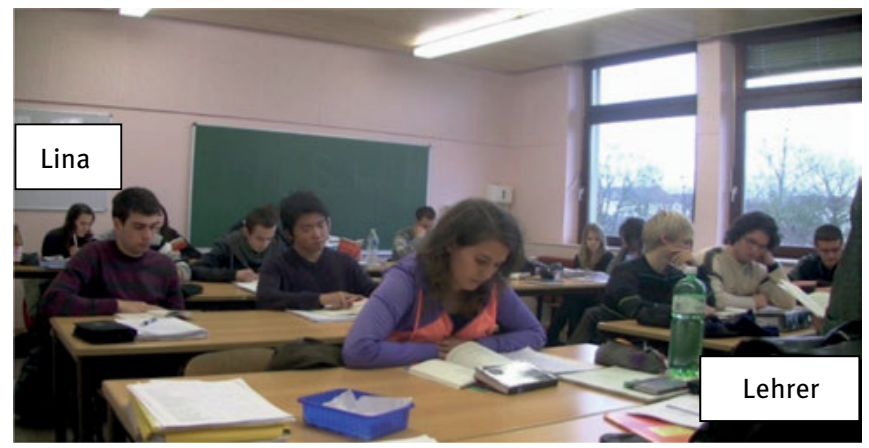

Bild 4: SchülerInnen zeigen durch Blickabwendung an, dass sie für Antwort nicht zur Verfügung stehen; Lehrer fordert auf: „lina FANG mal an,“.

Nachdem keine Wortmeldung erfolgt, senkt der Lehrer das Anspruchsniveau und kündigt (adhortativ, 08) an, dass die Aufgabe gemeinsam und in kleineren Schritten („STROphe für STROphe“, 09) angegangen werden soll. Als wieder keine Meldung erfolgt, fordert er die Schülerin Lina zum Vorlesen des Texts auf (11-12), wobei mal reflektiert, dass (auch) Lina keine besondere Bereitschaft für die Aufgabe signalisiert hatte. Auch hier wird der Gegenstand der Handlung, das Gedicht (vgl. 03), in der Imperativkonstruktion nicht erneut benannt.

Die bisher diskutierten Fälle zeigen zahlreiche Gemeinsamkeiten. Die aufgeforderte Handlung ist aufgrund des zuvor initiierten joint project erwartbar als Schritt zu seiner Realisierung. Die Handlung, zu der aufgefordert wird, kann jeweils problemlos vom Adressaten in der Situation vollzogen werden. Es handelt sich um eine Routinehandlung, die fraglos zum Handlungsrepertoire des Adressaten und zu den Pflichten bzw. Verantwortlichkeiten gehört, die (im Rahmen des joint project) mit seiner situativen Rolle bzw. Kategorienmitgliedschaft assoziiert sind. In den Imperativturns wird öfters das Objekt ausgelassen, da dieses aufgrund des bereits initiierten joint project bereits salient ist und fast immer auch schon zuvor benannt wurde. Das Objekt der Aufforderung ist für die Beteiligten entweder bereits wechselseitig im Fokus oder zumindest visuell verfügbar oder aufgrund von geteiltem Wissen identifizierbar. Die Verwendung von mal verweist darauf, dass der Adressat im Moment der Aufforderung auf diese leiblich, kogni- 
tiv oder hinsichtlich seiner Handlungserwartungen nicht vororientiert ist, wobei aber trotzdem die oben gegebenen Bestimmungen gelten.

\subsection{Insistieren auf einer vorangegangenen Aufforderung}

Das Insistieren auf einer vorangegangenen Aufforderung findet in der dritten (oder einer späteren) Sequenzposition in Bezug auf eine erste Aufforderung statt (Franke 1983). Das sequenzielle Muster sieht somit so aus:

1-A: Aufforderung (zumeist deklarativ, interrogativ oder durch deontischen Infinitiv),

2-B: Aufforderung wird nicht (kontinuierlich) befolgt,

3-A: Insistieren auf Aufforderung durch Imperativ.

Im Ausschnitt (4), wiederum aus einer Unterrichtsinteraktion, kündigt der Lehrer an, dass das joint project ,Sammeln von Lösungsvorschlägen“ fortgesetzt wird (01-02).

\#4 FOLK_127_23:49-23:59 Deutschunterricht im Wirtschaftsgymnasium

01 LE wir sammeln, (.)

02 wir sammeln WEIter.

$03 \quad(2.1)$

04 LE nina,

$05 \quad(0.5)$

06 LE he?

$07 \quad(0.2)$

08 LE was hast du AUFgeschrieben;

09 LE komm \#SAG_s mir;

10 bitte,

11 NI ich hab bei dem ZWEIte mehr aufgschriebe;

Als keine Meldungen der SchülerInnen erfolgen (03), teilt der Lehrer qua Namensnennung der Schülerin Nina die Aufgabe zu, weitere Vorschläge zu liefern (05). Doch auch nachdem er mit einer Partikel („he?“, 06) die bislang ausgebliebene Reaktion einfordert (vgl. Stivers/Rossano 2010), reagiert Nina nicht. Der Lehrer richtet daraufhin explizit an sie die Frage, was sie aufgeschrieben habe (08) und fordert sie imperativisch zur Nennung der von ihr aufgeschriebenen Lösungen auf (,komm SAG_s mir;“, 09). Der Imperativ wird hier also nach drei vorangegangenen erfolglosen Initiativen mit anderen sprachlichen Formen als Insistenzmittel benutzt. 
Auch im folgenden Fall aus einer praktischen Fahrschulstunde wird der Imperativ zum Insistieren auf einer zuvor bereits (hier deklarativ) formulierten Aufforderung eingesetzt:

\#5 FAHR_02_15_34:24-34:38 Praktische Fahrstunde

$01 \mathrm{FL}$ jetzt guckste wieder nach HINten \#und lenkst FERtig.

\#Bild 5

$02 \quad(0.6)$

$03 \mathrm{FL}$ und zwar woHIN?

$04 \quad$ (2.5)

$05 \mathrm{FL}$ wo soll dein AUto,=

06 =guck \#mal nach [HINten;]

$06 \mathrm{FS}$

[in DIE ]richtung.

\section{\#Bild 6}

$07 \mathrm{FL}$ guck mal nach HINten;

08 Wo soll dein Auto hin;

09 nach DA,

10 oder nach [DA; ]

11 FS [nach] DA.

Im Unterschied zu \#4 geht es in \#5 nicht darum, dass eine eingeforderte Reaktion ausgeblieben war, sondern darum, dass sie nicht hinreichend lange ausgeführt wurde. Die Schülerin hatte zunächst nach hinten geschaut (Bild 5), dann aber offenbar unter dem Eindruck der weiteren Aufforderung zu lenken (01) wieder nach vorn geschaut (Bild 6).

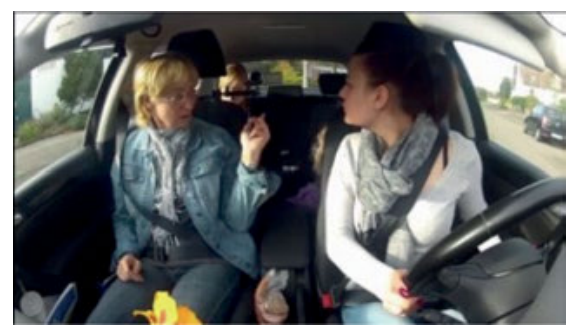

Bild 5: Fahrschülerin schaut nach hinten nach erster Aufforderung.

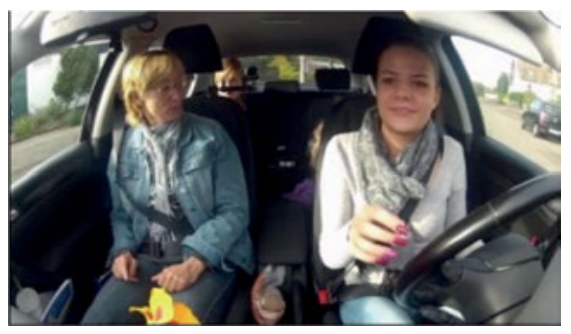

Bild 6: Fahrschülerin schaut nach vorn, als Fahrlehrerin insistiert: „guck mal nach HINten.“.

Der Imperativ signalisiert also in diesem Falle, dass die vorangegangene Aufforderung weiterhin zu befolgen ist. Das Beispiel zeigt, welche Schwierigkeiten 
bei der Befolgung von Aufforderungen entstehen können, wenn zugleich mehrere Aktivitätsstränge (Multiaktivität, s. Haddington et al. 2014) bearbeitet werden müssen. Die Verwendung von mal trägt der Tatsache Rechnung, dass die Fahrschülerin momentan mit dem Lenken beschäftigt ist.

\subsection{Korrektur einer Adressatenhandlung}

Imperative werden häufig für korrektive Instruktionen benutzt (Deppermann 2018a). Bei korrektiven Instruktionen wird der Adressat entweder zur Unterlassung oder Korrektur eines laufenden oder gerade vollzogenen Handelns aufgefordert, oder umgekehrt angewiesen, eine Handlung zu vollziehen, die aktuell (über)fällig ist, jedoch zu vollziehen versäumt wurde. Ein Beispiel für die Aufforderung zum Unterlassen einer Handlung findet sich in \#6 aus einer Fahrstunde. Als sich das Fahrschulfahrzeug einer Rechts-vor-links-Kreuzung nähert, an der es Vorfahrt hat, schaut der Fahrschüler nach links [Bild 7], offenbar um zu prüfen, ob Verkehr naht. Der Fahrlehrer fordert ihn daraufhin mit dem Imperativ „stopp- (.) <<h> STOPP,“ (08) zum Anhalten auf.

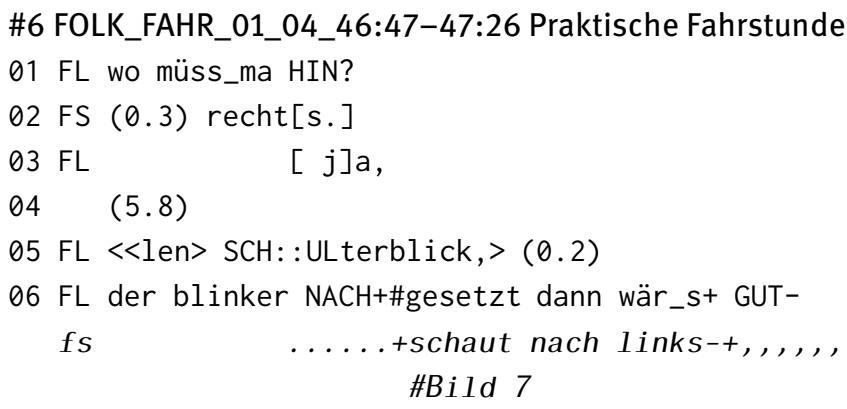

$07 \quad(0.7)$

$08 \mathrm{FL}{ }^{\circ} \mathrm{h}$ (.) stopp- (.) \#<<h> STOPP, >

\#Bild 8

09 FS (.) ja?

$10 \mathrm{FL}<<\mathrm{t}>$ WELche regel $>$ gilt hier?

11 FS RECHTS vor LINKS;

12 FL warum guckst du dann no_mal na_LINKS?

Das Anhalten des Fahrzeugs dient hier dazu, dem Fahrlehrer die Gelegenheit zu einer grundsätzlichen Erinnerung bzw. Belehrung über die Verhaltensregeln an Rechts-vor-links-Kreuzungen zu verschaffen, die beinhaltet, dass nicht nach links geschaut werden soll, wenn man Vorfahrt hat. Der Imperativ stopp wird hier wie 
in allen 108 Fällen im Korpus ohne Objekt benutzt ${ }^{6}$ - was gestoppt werden soll, ist für die Beteiligten aufgrund der perzeptuellen und pragmatischen Salienz relevanter Handlungen und Objekte evident und muss nicht expliziert werden.

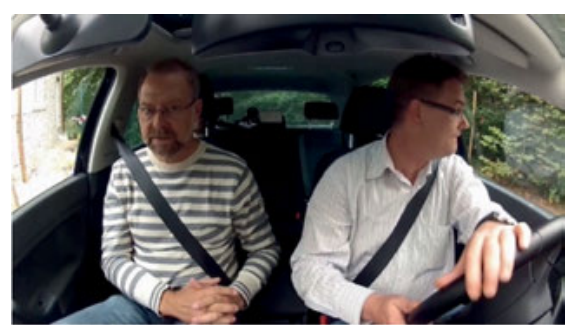

Bild 7: Fahrschüler schaut nach links vor Einbiegen in neue Straße.

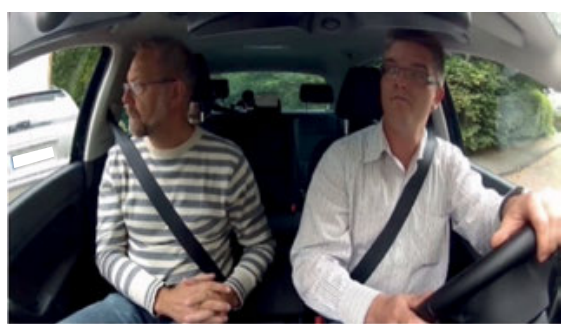

Bild 8: Fahrlehrer fordert Fahrschüler auf: „STOPP“ bei Einfahrt in neue Straße.

Während in \#6 eine kurz zuvor stattgefundene Handlung des Adressaten korrigiert wird, wird im nächsten Ausschnitt \#7 der Adressat im ersten Fall dazu aufgefordert, eine laufende Handlung zu suspendieren; im zweiten Fall dient der Imperativ dazu, eine antizipierte Handlung zu unterbinden. Zu Beginn der Gesprächsaufnahme besprechen S1 (der zu den Aufgenommenen zählt) und der Forscher (FO), was beim Umgang mit dem Mikrofon zu beachten ist. Nachdem der Forscher ermahnt hatte „NICHT gegen den tisch ditschen“ (04), interveniert S1 in dessen erkennbar nicht abgeschlossenen Turn mit dem Imperativ „WARte mal“ (07) und greift nach der Tischplatte (Bild 9).

\#7 FOLK_00039_00:30-00:46 Informelle Alltagsinteraktion

$01 \mathrm{~S} 1$ beim HUsten bisschen weiter zurück, =wa?

$02(0.3)$

03 FO ja geNAU,

04 und NICHT gegen den tisch ditschen irgendwie;

05 also ich hab da schon DIE-

$06 \quad(0.6)$

07 S1 \%WARte \#mal;

s1 \%..........

\#Bild 9

08 F0 die schaumstoffma\%tte; (.)

s1 ..........\%greift Tischplatte---->

6 In nur zwei Fällen findet sich eine Ortsbestimmung, wo gestoppt werden soll. In allen anderen Fällen wird sofortiger Vollzug erwartet. 
09 FO naja stell_s NICH so weit weg.

10 das schon oKAY so.

$11(0.1) \%(0.3)$

s1---- \%

12 s1 jut.

Mit „WARte mal“ (07) fordert S1 FO auf, seinen laufenden Turn abzubrechen mal zeigt hier an, dass für FO an dieser Stelle die Intervention nicht zu erwarten ist. Wie bei stopp wird auch bei warte so gut wie nie expliziert, worauf bzw. womit gewartet werden soll. Es bezieht sich stets auf das laufende bzw. als nächstes antizipierbare Adressatenhandeln. Gleichzeitig greift S1 nach der Tischplatte (Bild 9), was FO als Handlungsabsicht versteht, dass S1 den Tisch von der Sitzgruppe, auf der die Aufzunehmenden sitzen, wegrücken will. Offenbar hatte S1 aus FOs Aufforderung in 04 die Schlussfolgerung gezogen, dass es besser sei, den Tisch weiter wegzurücken.

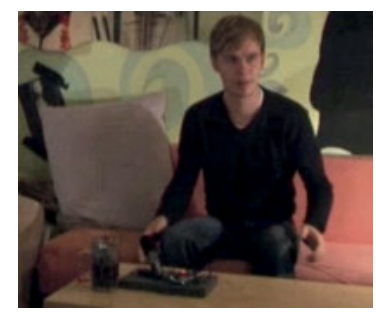

Bild 9: S1 greift zum Tisch, als er „WARte mal;“ zu FO (außerhalb des Bilds) sagt.

Diese antizipierte Handlung von S1 korrigiert FO in 09 mit dem Imperativ ,stell_s NICH so weit weg“. S1 nimmt schließlich die Hände von der Tischplatte, ohne den Tisch bewegt zu haben (11). Beide Sprecher reagieren also antizipativ auf erschlossene (Handlungs-)Intentionen ihres Partners.

Korrektive Aufforderungen können nicht nur wie in \#6 und \#7 zum Unterlassen einer Handlung auffordern. Sie können auch zu einem alternativen Handeln auffordern oder wie in \#8 aus einer Instruktion bestehen, wie die fragliche Handlung korrekt(er) auszuführen ist. Vor \#8 hatte der Einsatzleiter (EL) seinen Assistenten (AS) gebeten, ihm eine Armschiene für die Anbringung am Patienten vorzubereiten. AS bringt zunächst eine Gelenkschiene, die EL für ungeeignet hält (01-07). Nachdem EL präzisiert hat, was für eine Schiene er haben will (11), wendet sich AS ab, um eine andere zu holen (12). In diesem Moment fordert EL AS auf, ihm die Gelenkschiene zu geben (13-15). 


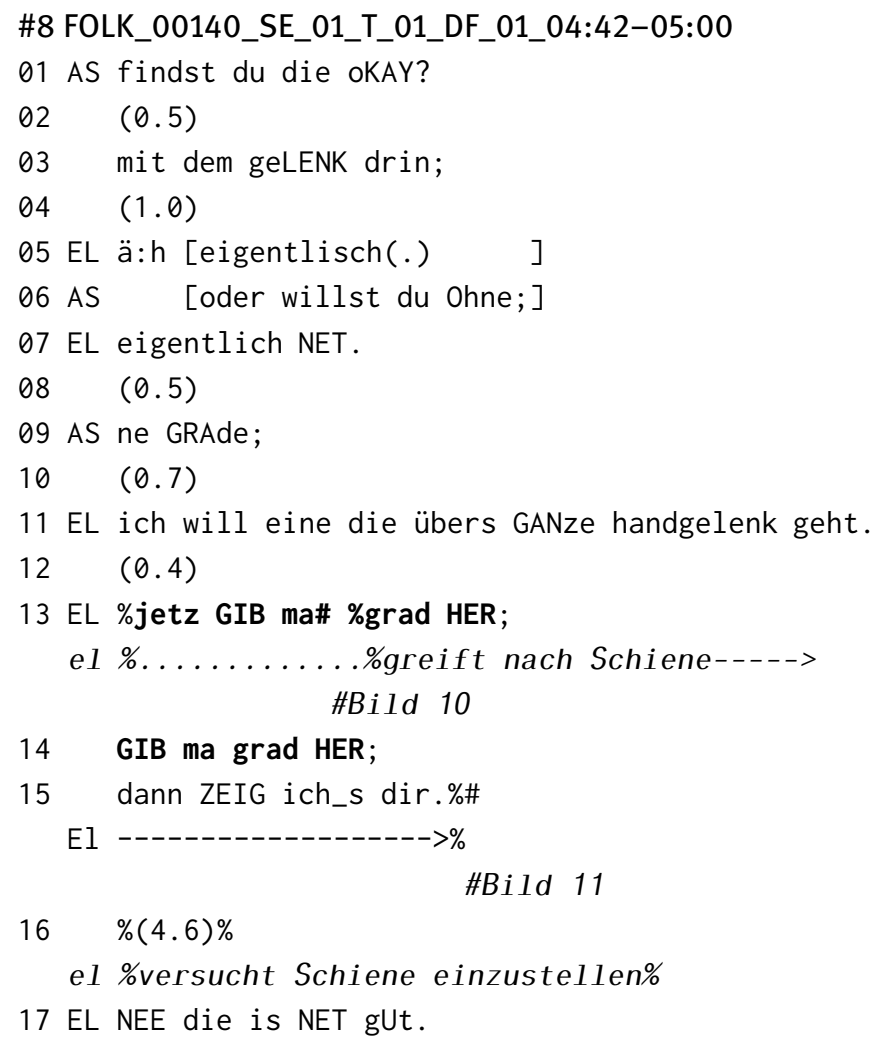

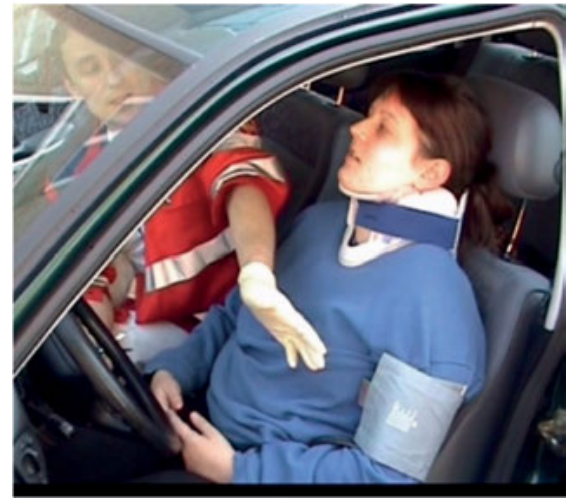

Bild 10: EL streckt Hand nach Schiene aus und fordert AS auf: „gib mal grad HER,“.

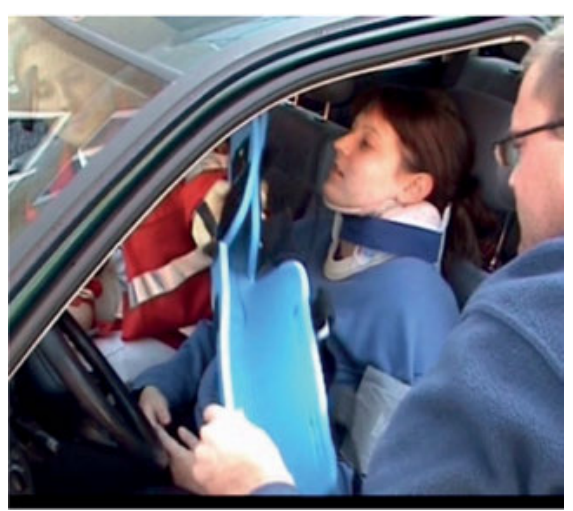

Bild 11: EL ergreift Schiene, die ihm AS reicht. 
Mit den Imperativen ,jetz GIB ma grad her; GIB ma grad her“ (13-14, Bild 10-11) unterbricht EL den von AS begonnenen Handlungsablauf. Die Korrektur unterbricht das aktuelle Handeln des Adressaten, bezieht sich aber auf ein früheres Handeln des Adressaten. EL versucht AS zu demonstrieren, wie die Schiene besser einzustellen ist, als dies AS gelungen war (16); er stellt aber fest, dass dies mit dieser Art von Schiene nicht möglich ist (17).

Das Ausbleiben eines in der momentanen Situation gebotenen Handelns ist eine weitere Motivation für korrektive Aufforderungen. Derartige Instruktionen sind vor allem in den Fahrschuldaten besonders häufig. In \#9 ist das Fahrschulauto gerade in eine Straße eingebogen. Eine grundsätzliche Anforderung in dieser Situation besteht darin, Verkehrszeichen und Fahrbahnmarkierungen daraufhin zu prüfen, auf welche Spur sich die Fahrschülerin einordnen muss, um in einer bestimmten Richtung weiterfahren zu können. Wenn der Fahrlehrer keine Richtungsinstruktion gibt, muss die Fahrschülerin stets geradeaus fahren, sofern dies möglich ist. In \#9 muss sie dazu auf die linke Spur wechseln. ${ }^{7}$

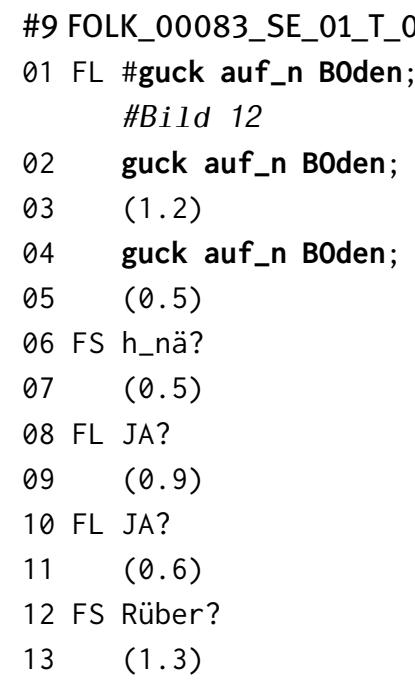

Als die Fahrschülerin in die neue Straße einbiegt, nimmt sie die rechte Spur. Der Fahrlehrer behandelt diese Fehlentscheidung als Resultat des Versäumnisses der Fahrschülerin, nicht auf die Fahrbahnmarkierungen zu schauen (Bild 12).

Der Fahrlehrer fordert sie nicht direkt dazu auf, die Spur zu wechseln, sondern produziert dreimal eine korrektive Instruktion im Imperativ ,guck auf_n BO-

7 Dieser Ausschnitt wird in Deppermann (2015) in Bezug auf die Verständigungs- und Kooperationsprobleme zwischen Fahrlehrer und -schülerin untersucht. 


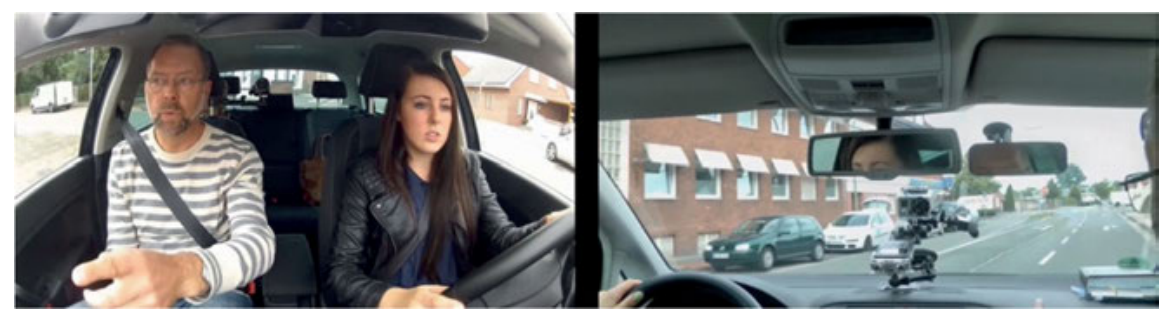

Bild 12: Fahrlehrer zeigt auf Fahrbahnmarkierungen und fordert Schülerin auf: „guck auf den BOden;“

den;“ (01-04; mit Zeigegeste, Bild 12). Die Anweisung fordert die versäumte Handlung ein. Zugleich verbindet sich mit ihr die Erwartung, dass die Fahrschülerin aus dem, was sie auf dem Boden sehen kann - die Fahrbahnmarkierungen -, die gewünschte Konsequenz des Spurwechsels zieht. Für die entsprechende Inferenz sucht die Schülerin dann auch in 12 die Bestätigung des Fahrlehrers.

Imperative, mit denen ein Handeln des Adressaten korrigiert wird, können also in unterschiedlichen Zeitverhältnissen zur korrektiven Aufforderung stehen: Das zu korrigierende Handeln kann eben gerade abgeschlossen worden sein, es kann andauern oder es kann als nächste Handlung des Adressaten, z. B. aufgrund bereits stattfindender Präparation der Handlung, antizipiert werden. Korrektive Aufforderungen können sich auch auf das Ausbleiben fälliger Handlungen des Adressaten beziehen. Korrektive Aufforderungen per Imperativ sind als Erinnerungen gestaltet: Ihre knappe, wenig explizite Formulierung (mit reduzierter Argumentstruktur) indiziert common ground und damit, dass dem Adressaten die korrekte Handlungsweise grundsätzlich bekannt ist (Deppermann 2018a). Ihre situierte Interpretation verdankt sich wesentlich dem Bezug auf das übergeordnete joint project und auf visuell Verfügbares. Schnelle Wiederholungen des Imperativs (wie in \#6, \#8 und \#9) kontextualisieren in besonderem Maße die Dringlichkeit der sofortigen Befolgung der Aufforderung.

\subsection{Ratifikation einer Partnerinitiative bzw. Erlaubnis}

Imperative können benutzt werden, um eine unmittelbar vorangegangene Initiative des Partners zu ratifizieren (vgl. fürs Finnische Sorjonen 2017). Der Partner kann dabei explizit um die Erlaubnis für eine geplante Handlung gebeten haben. In \#10 fragt bspw. ein Rettungsassistent (AS1), ob er den Notarzt rufen soll (01). Der Einsatzleiter erteilt mit zwei Imperativen die Erlaubnis: 
\#10 FOLK_00138_SE_01_T_01_DF_01_01.11-01.17 Rettungssanitäterübung

01 AS1 soll ich gleich mal_n NOTarzt noch nachfordern,

02 EL JA das kannst du gleich machen;

03 geh ans AUto,

04 hol_n NOTarzt,

05 so wir drehn jetzt UM,

$06 \quad(0.4)$

07 EL alles klar.

Auch wenn der Partner eigene Handlungen ankündigt oder beschreibt, kann der Imperativ zum Ausdruck der Zustimmung bzw. der Erlaubnis verwendet werden. Letzteres gilt, wenn es sich um Handlungen handelt, über deren Legitimität zu entscheiden der Imperativ-Sprecher die deontische Autorität hat oder die ihn inkommodieren könnten. Dies ist in \#11 der Fall. Gast LH bemerkt, dass sie als Einzige die zur freien Bedienung aufgestellten Mirabellen isst (01-02). Sie markiert damit, dass ihr bewusst ist, dass man ihr Verhalten als unhöflich auffassen könnte, gibt aber implizit auch zu verstehen, dass die TischgenossInnen die Gelegenheit sich auch zu bedienen nicht wahrnehmen und daher mitverantwortlich für das entstehende Ungleichgewicht sind.

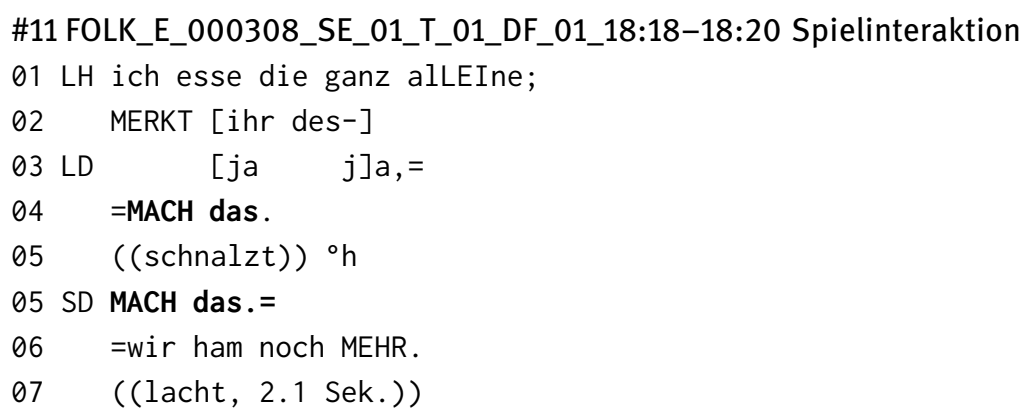

Beide GastgeberInnen (LD und SD) erteilen mit dem Imperativ „MACH das.“ LH explizit die Erlaubnis (alleine) weiter zu essen. SD liefert noch eine Begründung nach, die LHs Verhalten weiter entproblematisiert, da sie das potenzielle moralische Problem, dass LH den anderen etwas wegessen könnte, für gegenstandslos erklärt.

Ratifikationen bzw. Erlaubnisse sind im Unterschied zu allen anderen Imperativverwendungen primär responsiv und nicht initiativ. Sie sind second pair parts zu vorangehenden Handlungen wie Vorschlägen, Angeboten, Bitten um Erlaubnis. Sie machen Zustimmung, Bestätigung, Erlaubnis etc. relevant. Die deontische Autorität des Imperativ-Sprechers wird dabei oft schon durch das Ersuchen um 
Bestätigung etc. vom Adressaten angezeigt oder auch durch Letzteren selbst verdeutlicht (wie implizit in \#11, 06 durch SDs Begründung).

\subsection{Imperative zur Initiierung neuer joint projects}

Die bisherigen Analysen haben gezeigt, dass Imperative im Kontext von bereits etablierten joint projects benutzt werden und dort jeweils sequenziell erwartbare Handlungen beauftragen bzw. ratifizieren. In seltenen Fällen werden in den untersuchten Daten Imperative aber auch benutzt, um ein neues joint project zu eröffnen. Dass es sich dabei um eine grundsätzlich andere Verwendung handelt, zeigt sich an der Gestaltung der Imperativ-Turns. Im Unterschied zu den bisher betrachteten sind sie erheblich elaborierter. Vor allem werden sie aufwändig modalisiert, wie dies für Bitt- und Aufforderungshandlungen, deren Legitimität oder Zumutungscharakter für den Sprecher fraglich erscheint, in anderen deklarativen oder interrogativen Formaten gängig (vgl. Brown/Levinson 1987: 174-176), aber sonst bei Imperativen ganz unüblich ist.

Ein Beispiel ist \#12. Hier hat der Einsatzleiter eben ein project abgeschlossen (01-02). Er ist gerade dabei, sich wieder der Patientin, die im Auto sitzt, zuzuwenden. AS passt diesen Moment ab, um EL um einen Rat zu bitten (03-05).

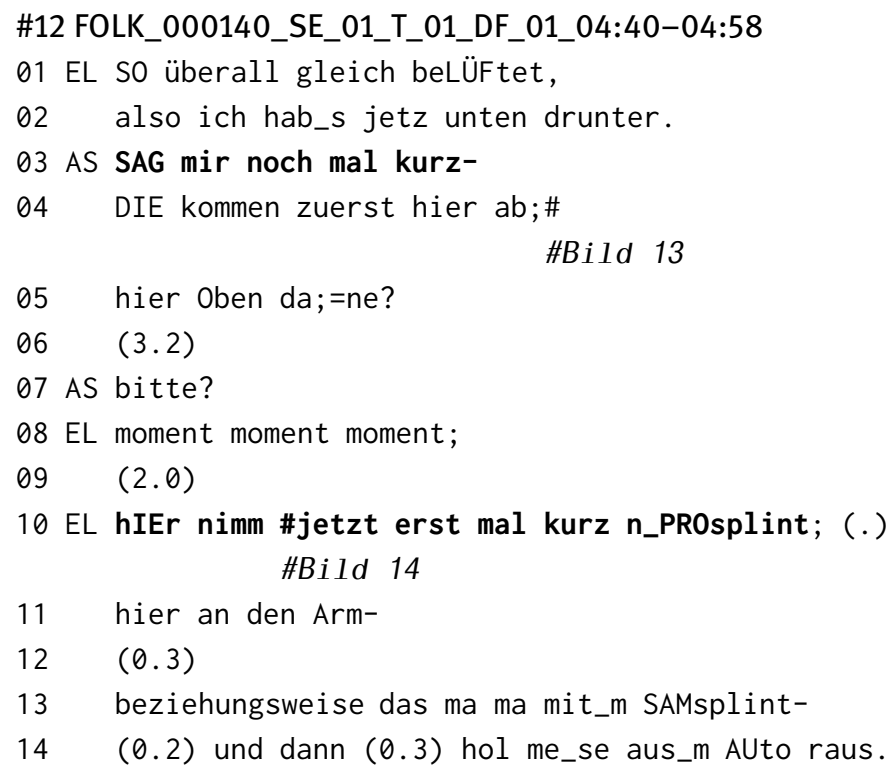


AS formuliert seine Bitte mithilfe einer Imperativ-Projektorkonstruktion (zu Projektorkonstruktionen s. Günthner 2008). Die Konstruktion ist aufwändiger als in den bisher betrachteten Fällen: Die Bitte wird in mehreren TCUs realisiert, die Imperativ-TCU selbst ist durch Modalpartikel und Zeitangabe (,noch mal kurz“, 03), das indirekte Objekt „mir“ wird formuliert. Der Sprecher zeigt mit „mal“ an, dass er davon ausgeht, dass der Adressat EL nicht auf die Bitte vorbereitet ist. Er ist darum bemüht, ihren Zumutungscharakter für den Adressaten zu minimieren („kurz“). Während AS seine Bitte formuliert, wendet sich EL bereits wieder der Patientin im Auto zu (Bild 13), ist also offenbar auf einen anderen Handlungszusammenhang orientiert. AS' Vorsicht bei der Formulierung seiner Bitte scheint nur allzu berechtigt, denn EL geht nicht auf sie ein. EL richtet, nach der Anzeige, dass das von AS initiierte neue project im Moment nicht weiterverfolgt werden soll („moment moment moment;“, 08), stattdessen selbst eine Aufforderung an AS (10, Bild 14).

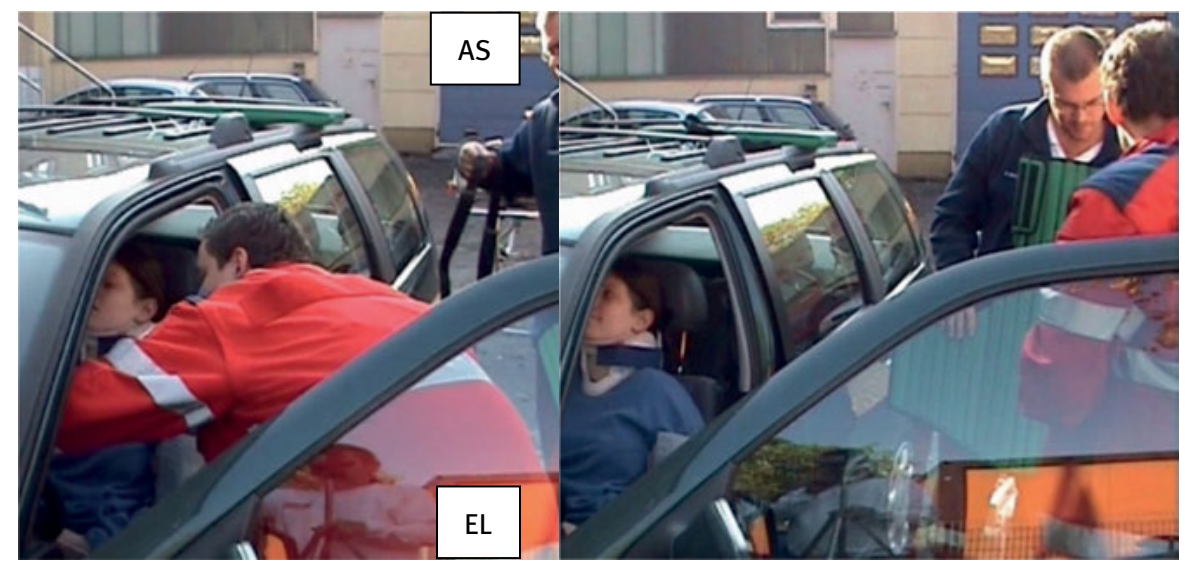

Bild 13: AS fordert EL auf, ihm bei der Einrichtung der Trage zu helfen.
Bild 14: EL fordert AS auf einen Prosplint zu holen.

Wie zuvor AS in 03 benutzt auch EL in 10 Modalpartikel und Zeitangaben (,jetzt erst mal kurz“, 10), die anzeigen, dass er erwartet, dass der Adressat nicht auf die Aufforderung vorbereitet ist („mal“) und dass er zugleich den Zumutungscharakter zu minimieren sucht (,kurz“). Mit dem Turn-initialen „hIEr“, das einen neuen Aufmerksamkeitsfokus ankündigt, wird ein neues joint project eröffnet, auf das AS nicht vorbereitet ist und das in diesem Fall sogar dessen Handlungsintentionen zuwiderläuft. Auch das Objekt „PROsplint“ muss explizit gemacht werden, da 
es sich nicht im Gesichtsfeld der Beteiligten befindet und in der vorangegangenen Sequenz nicht salient war.

Die sprachliche Gestaltung von Imperativen, mit denen neue joint projects eröffnet werden, unterscheidet sich grundlegend von derjenigen, die bereits eröffnete joint projects fortschreiben und auf die Verfügbarkeit und konsonante Erwartungen des Adressaten setzen können. Objekte werden explizit lexikalisch formuliert, da nicht auf bereits visuell und/oder pragmatisch salienten Objektbezügen aufgebaut werden kann. Die Imperativ-Turns werden durch zeitliche, lokale, direktionale und umstandsbezogene Adverbiale näher bestimmt und durch Modalpartikeln abgetönt. Insgesamt sind sie daher sprachlich deutlich komplexer. Der Sprecher zeigt damit, dass ihm bewusst ist, dass der Adressat nicht fraglos für die beauftragte Handlung bereitsteht, nicht auf sie vororientiert ist und manchmal gar einen konkurrierenden Handlungsplan verfolgt. Dennoch werden auch zur Eröffnung neuer joint projects nur dann Imperative eingesetzt, wenn - wie in den anderen Fällen - die beauftragte Handlung einfach und problemlos für den Adressaten zu vollziehen ist und keiner weiteren Erläuterung bedarf.

\section{Zusammenfassung der Ergebnisse zur Verwendung von Imperativen im Deutschen}

Die Verwendung von Imperativen ist eng an die vorgängige Etablierung eines joint project von SprecherInnen und AdressateInnen gebunden. Dabei hat der Imperativsprecher fast immer eine deontische und epistemische Autorität (z. B. als Vorgesetzter, Lehrer oder Gastgeber) inne, aus der die Aufforderungshandlung legitimiert ist. Diese Autorität bedeutet kaum einmal Willkür: Mit den Imperativen in unserem Korpus wird fast immer $\mathrm{zu}$ Handlungen aufgefordert, die in der Verantwortlichkeit und Zuständigkeit des Adressaten liegen, welche sich ihrerseits aus kanonischen Handlungsabläufen und Arbeitsteilung ergeben. ${ }^{8}$ Mit Imperativen werden einfache Routinehandlungen beauftragt, die keiner Erklärung bedürfen. Da Imperative laufende, häufig objektzentrierte joint projects

\footnotetext{
8 Im FOLK-Korpus finden sich natürlich auch Verwendungen, die nicht in dieser Weise legitimiert sind. Sie sind aber sehr selten. Z. B. kommt das Format [halt das Maul/die Klappe/die Fresse/die Gosch] 15 Mal vor. Es wird in den untersuchten Daten zwischen Freunden und Familienmitgliedern benutzt, zumeist in Spielkontexten und in einer Art derben Vertrautheit, ähnlich einer rituellen Beschimpfung. Nicht betrachtet wurde hier auch die Verwendung von Imperativen in eskalierenden Streits. Entsprechende Daten fehlen in FOLK. Die Untersuchung dieser beiden Verwendungskontexte stellt ein Desiderat für weitere Untersuchungen dar.
} 
fortschreiben bzw. visuell verfügbare Gegenstände betreffen, können sie mit einer schlanken Syntax (Objekttilgung, keine adverbialen Bestimmungen) realisiert werden. Stattdessen werden manchmal Zeigegesten eingesetzt. Dies ist aber generell üblich für die Referenz auf anwesende Gegenstände (s. Stukenbrock 2015 und Stukenbrock i.d. B.) und nicht spezifisch für Imperative. Imperative sind aktualistische Aufforderungsformate: Sie fordern zu sofortiger, manchmal gar dringender Befolgung auf und reagieren oft auf unmittelbar im gemeinsamen Handlungsprozess entstehende Kontingenzen (wie laufendes oder unmittelbar vorangehendes Adressatenhandeln, Veränderungen in der räumlichen Umgebung, nächste anstehende Schritte eines Handlungsablaufs). Dabei kann der genaue Zeitbezug zum Handeln des Adressaten (reaktiv, gleichzeitig, antizipatorisch) variieren. Hat der Imperativ-Turn ein nicht-minimales Format, zeigt der Sprecher damit an, dass er annimmt, dass der Adressat auf die Handlung, zu der aufgefordert werden soll, noch nicht orientiert ist. In Bezug auf diesen Parameter, Vororientierung des Adressaten auf die Aufforderung, gibt es Abstufungen und unterschiedliche Fälle. Im Einklang mit den Annahmen der accessibility scale (Ariel 1990, 2008) zeigt die Nichtrealisierung des Objekts an, dass der Gegenstand der beauftragten Handlung pragmatisch und/oder visuell für den Adressaten salient ist. Gleiches gilt für klitische oder pronominale Objekte, die aber bemerkenswerterweise seltener als Nullrealisierungen sind, was ebenfalls den hochgradig erwartbaren Charakter der Handlung unterstreicht. Lexikalische Objektrealisierungen sind selten und zeigen an, dass es sich um eine lokal nicht erwartete Aufforderung handelt und/oder ein nicht visuell verfügbarer bzw. zuvor nicht fokussierter Gegenstand benötigt wird. Hinsichtlich der angenommenen Vororientierung des Adressaten auf die Aufforderung machen Sprecher feine Unterschiede: Modalisierung des Imperativturns mit mal zeigt an, dass der Adressat wohl als grundsätzlich für die Aufforderung verfügbar betrachtet wird, im Augenblick diese aber nicht erwartet, anderweitig involviert ist oder körperlich anders ausgerichtet ist. Umfänglichere Modalisierungen und weitere spezifizierende und explizierende Elemente im Imperativturn, wie z. B. die explizite Handlungskategorisierung der erwarteten Reaktion durch eine vorgeschaltete Projektorkonstruktion, modale, temporale, lokale, direktionale, instrumentale etc. Adverbiale und Attribute, zeigen an, dass kein joint project fortgesetzt wird, sondern zu einer lokal nicht erwartbaren oder gar mit anderen projects konkurrierenden Handlung aufgefordert wird, für die die Kooperation des Adressaten nicht unbedingt sicher erscheint. 


\section{Theoretische Diskussion der Ergebnisse}

Wir haben uns bei den obigen Analysen der terminlogischen Entscheidung enthalten, um was für Strukturen es sich bei den untersuchten Imperativverwendungen handelt. Wir wollen nun die Befunde in Bezug auf die eingangs skizzierten drei theoretischen Alternativen diskutieren: grammatische Konstruktionen, sprachliche Praktiken und social action formats.

Die identifizierten Imperativverwendungen können allesamt als Fälle einer unterspezifizierten grammatischen Konstruktion modelliert werden. Ihre Formseite besteht aus der morphologischen Form des Imperativs und der topologischen V1-Position. Ihre Inhaltsseite beinhaltet eine unterspezifizierte deontische Handlungsfunktion, die anzeigt, dass die Handlung sofort ausgeführt werden soll. Dies umfasst Bitten, Instruktionen, Aufforderungen, Ratifikationen, Erlaubnisse etc., welche aber auf der Ebene der konstruktionsgrammatischen Analyse nicht zu differenzieren sind. Diese generische Imperativkonstruktion ist indifferent gegenüber spezifischen Kontextbedingungen. An ihre Seite tritt die Konstruktion [Imperativ + mal]. In Bezug auf die Inhaltsseite unterscheidet sie sich nicht von der generischen Konstruktion, präsupponiert aber eine wichtige schematische Kontextvoraussetzung, nämlich dass der Adressat zwar verfügbar, aber nicht vorbereitet ist auf die Aufforderung.

Die in dieser Untersuchung identifizierten fünf Varianten der Verwendung von Imperativen sind dagegen $\mathrm{m}$. E. nicht als unterschiedliche Konstruktionen, sondern als verschiedene Praktiken zu verstehen. Die fünf Verwendungen sind jeweils an eine ganz spezifische sequenzielle Umgebung gebunden und spezifizieren mit Bezug auf diese Umgebung die allgemeine Aufforderungsfunktion. Damit ist gemeint, dass die Handlungsfunktion nicht unabhängig vom sequenziellen (Prä-)Kontext beschreibbar ist: Eine korrektive Instruktion setzt bspw. ein Fehlverhalten des Adressaten voraus, eine Ratifikation eine vorangegangene Initiative etc. Solche Verhältnisse in eine Konstruktionsbeschreibung integrieren zu wollen, geht weit über das Spektrum von konstruktionscharakterisierenden Eigenschaften hinaus und erfordert andere Analyseinstrumentarien als die der Konstruktionsgrammatik. Bei diesen Praktiken verhält es sich gewissermaßen umgekehrt zur Konstruktion [Imperativ + mal]: Letztere erfordert von sich aus einen spezifischen Kontext, ist aber ansonsten eine typische first pair part-Konstruktion, die als solche zu ihrer generischen Definition keinen Präkontext erfordert. Tatsächlich aber wird jeder Imperativ-Turn in einem bestimmten Kontext produziert. So wie die mit dem Imperativ realisierte Handlung selbst ein Verständnis dieses Kontexts anzeigt bzw. auf ihm aufbaut (z. B. dass ein joint project bereits im Gange ist), so ist sie umgekehrt in ihrer Interpretation als spezifische Art von deontischer 
Handlung von diesem Kontext abhängig und kann die spezifische Interpretation in der Regel nicht aus sich selbst gewährleisten. ${ }^{9}$ Für die situierten Verwendungsbedingungen spielen ebenso wie für die Handlungsinterpretation, zusätzlich zur Grammatik, Lexikalisierung und körperliche Konstellationen und Aktivitäten eine Rolle. Der Imperativ ist also aus unserer Sicht am besten als Konstruktion zu verstehen, die sequenziell und leiblich sensitiv für bestimmte Praktiken als Ressource benutzt wird und in diesem Kontext indexikalisch weiter spezifiziert wird. Darin besteht ihre Flexibilität, die, wie die Fallbeispiele zeigen, offen für weitere kontextspezifischere Adaptationen sind. Wir wollen denn auch keineswegs behaupten, dass die hier dargestellten fünf Praktiken die Verwendungen des Imperativs im Deutschen erschöpfen. Wie in Fußnote 7 angesprochen, sind weitere Praktiken in anderen Interaktionskontexten zu erwarten. Dabei ist die Grenze zwischen generischer Praktik und fallspezifischer Realisierung je nach Erkenntnisinteresse und Menge und Ähnlichkeit der betrachteten einschlägigen Fälle flexibel.

Wir haben oben festgestellt, dass der Begriff social action format theoretisch kaum bestimmt ist. Wenn Sequenzkontext und leibliches Handeln in seine Definition einbezogen werden, dann nähert er sich dem Praktikenbegriff an (vgl. auch Linke i. d. B.). Im Unterschied zum Praktikenbegriff geht social action format allerdings nicht von einer holistischen Praktik aus, sondern von einer sprachlichen Form. Wir haben aber zeigen können, dass (auch) im Bereich der Imperative die genaue Handlungsfunktion nicht durch die sprachliche Form determiniert ist, sondern erst im Sequenzkontext klar wird. Ob bspw. etwas eine korrektive Instruktion ist oder ein nächster Routineschritt, hängt z. B. vom genauen Zeitpunkt der Aufforderung und vom aktuellen bzw. vorangegangenen Handeln des Adressaten ab. In Bezug auf die Debatte um die relative Bedeutung von Sequenz vs. sprachlichem Format für die Handlungskonstitution (s. als Gegenpositionen etwa Schegloff 1984 vs. Couper-Kuhlen 2014) können wir feststellen, dass im Falle der Imperative die sprachliche Konstruktion als solche wohl einen sehr grob bestimmten Handlungstyp vorgibt, die genaue lokale Handlungsfunktion aber von der sequenziellen Position abhängt (vgl. a. Clift 2016). Imperative gehören damit zu Praktiken einer „positionally sensitive grammar“ (Schegloff 1996), die allerdings auf einem grammatisch verbürgten Potenzial aufbauen und somit nur bedingt, aber essenziell, d. h. für ihre lokale indexikalische Interpretation erforderlich, flexibel sind.

\footnotetext{
9 Ausnahmen bestehen hier durch die Lexikalisierung. Z. B. zeigen stopp und warte immer an, dass es sich um eine korrektive Aufforderung handelt, die ein laufendes oder gerade abgelaufenes Partnerhandeln unterbinden soll.
} 
Interaktion. Untersuchungen zum Zusammenhang von grammatischen Strukturen und Gesprächsprozessen, S. 43-65. Radolfzell: Verlag für Gesprächsforschung.

Deppermann, Arnulf (2007): Grammatik und Semantik aus gesprächsanalytischer Sicht. Berlin: de Gruyter.

Deppermann, Arnulf (2011): Konstruktionsgrammatik und Interaktionale Linguistik: Affinitäten, Komplementaritäten und Diskrepanzen. In: Lasch, Alexander und Alexander Ziem (Hrsg.), Konstruktionsgrammatik III. Aktuelle Fragen und Lösungsansätze, S. 205-238. Tübingen: Stauffenburg.

Deppermann, Arnulf (2015): When recipient design fails: Egocentric turn-design of instructions in driving school lessons leading to breakdowns of intersubjectivity. In: Gesprächsforschung - Online-Zeitschrift zur verbalen Interaktion, 16:63-101.

Deppermann, Arnulf (2018a): Instruction practices in German driving lessons: Differential uses of declaratives and imperatives. In: International Journal of Applied Linguistics, 28(2):265-282.

Deppermann, Arnulf (2018b): Sprache in der multimodalen Interaktion. In: Deppermann, Arnulf und Silke Reineke (Hrsg.), Sprache im kommunikativen, interaktiven und kulturellen Kontext, S. 51-86. Berlin: de Gruyter.

Deppermann, Arnulf und Mechthild Elstermann (2008): Lexikalische Bedeutung oder Konstruktionsbedeutungen? Eine Untersuchung am Beispiel von Konstruktionen mit ,verstehen'. In: Stefanowitsch, Anatol und Kerstin Fischer (Hrsg.), Konstruktionsgrammatik II. Von der Konstruktion zur Grammatik, S. 103-133. Tübingen: Stauffenburg.

Deppermann, Arnulf, Helmuth Feilke und Angelika Linke (Hrsg.) (2016a): Sprachliche und kommunikative Praktiken. Jahrbuch des Instituts für Deutsche Sprache 2015. Berlin: de Gruyter.

Deppermann, Arnulf, Helmuth Feilke und Angelika Linke (2016b): Sprachliche und kommunikative Praktiken: Eine Annäherung aus linguistischer Sicht. In: Deppermann, Arnulf, Helmuth Feilke und Angelika Linke (Hrsg.), Sprachliche und kommunikative Praktiken. Jahrbuch des Instituts für Deutsche Sprache 2015, S. 1-23. Berlin: de Gruyter.

Deppermann, Arnulf und Susanne Günthner (2015): Introduction: Temporality in interaction. In: Deppermann, Arnulf und Susanne Günthner (Hrsg.), Temporality in Interaction, S. 1-23. Amsterdam: John Benjamins.

Donhauser, Karin (1986): Der Imperativ im Deutschen: Studien zur Syntax und Semantik des deutschen Modussystems. Hamburg: Buske.

Duden (2016): Die Grammatik. Berlin: Bibliographisches Institut.

Feilke, Helmuth (1996): Sprache als soziale Gestalt. Frankfurt a. Main: Suhrkamp.

Fischer, Kerstin (2010): Beyond the sentence: Constructions, frames and spoken interaction. In: Constructions and Frames, 2(2):185-207.

Fischer, Kerstin (2015): Conversation, Construction Grammar, and cognition. In: Language and Cognition, 7:563-588.

Fox, Barbara A. (2007): Principles shaping grammatical practices: an exploration. In: Discourse Studies, 9(3):299-318.

Fox, Barbara A. und Trine Heinemann (2016): Rethinking format: an examination of requests. In: Language in Society, 45(4):499-531.

Fox, Barbara A. und Robert Jaspersen (1995): A syntactic exploration of repair in English conversation. In: Davis, Philip W. (Hrsg.), Alternative Linguistics, S. 77-134. Amsterdam: John Benjamins.

Franke, Wilhelm (1983): Insistieren. Göppingen: Kümmerle. 
Goldberg, Adele (1995): Constructions. A construction grammar approach to argument structure. Chicago, IL: University of Chicago Press.

Goldberg, Adele (2003): Constructions: a new theoretical approach to language. In: Trends in Cognitive Sciences, 7(5):219-224.

Goldberg, Adele (2006): Constructions at Work: the nature of generalization in language. 0xford: OUP.

Goodwin, Marjorie Harness (2006): Participation, Affect, and Trajectory in Family Directive/ Response Sequences. In: Text and Talk, 26(4/5):513-542.

Goodwin, Marjorie Harness und Asta Cekaite (2013): Calibration in directive/response sequences in family interaction. In: Journal of Pragmatics, 46(1):122-138.

Günthner, Susanne (2006): Von Konstruktionen zu kommunikativen Gattungen: Die Relevanz sedimentierter Muster für die Ausführung kommunikativer Aufgaben. In: Deutsche Sprache, 34(1/2):173-190.

Günthner, Susanne (2008): Projektorkonstruktionen im Gespräch: Pseudoclefts, die Sache istKonstruktionen und Extrapositionen mit es. In: Gesprächsforschung - Online-Zeitschrift zur verbalen Interaktion, 9:86-114. Online verfügbar unter: www.gespraechsforschungonline.de/heft2008/ga-guenthner.pdf.

Günthner, Susanne (2010a): Konstruktionen in der kommunikativen Praxis - Zur Notwendigkeit einer interaktionalen Anreicherung konstruktionsgrammatischer Ansätze. In: Zeitschrift für Germanistische Linguistik, 37(3):402-426.

Günthner, Susanne (2010b): Sprache und Sprechen im Kontext kultureller Praktiken. Facetten einer Anthropologischen Linguistik. In: Meyer, Silke und Armin Owzar (Hrsg.), Disziplinen der Anthropologie, S. 121-144. Frankfurt: Waxmann.

Günthner, Susanne (2011): Konstruktionen in der gesprochenen Sprache. In: Habscheid, Stephan (Hrsg.), Textsorten, Handlungsmuster, Oberflächen. Linguistische Typologien der Kommunikation, S. 296-313. Berlin: de Gruyter.

Günthner, Susanne (2016): Praktiken erhöhter Dialogizität: Onymische Anredeformen als Gesten personifizierter Zuwendung. In: Zeitschrift für Germanistische Linguistik, 44(3):406-436.

Günthner, Susanne (2017): Diskursmarker in der Interaktion - Formen und Funktionen univerbierter guck mal- und weißt du-Konstruktionen. In: Blühdorn, Hardarik et al. (Hrsg.), Diskursmarker im Deutschen. Reflexionen und Analysen, S. 103-130. Göttingen: Verlag für Gesprächsforschung.

Günthner, Susanne (2018): Routinisierte Muster in der Interaktion. In: Deppermann, Arnulf und Silke Reineke (Hrsg.), Sprache im kommunikativen, interaktiven und kulturellen Kontext, S. 29-50. Berlin: de Gruyter.

Günthner, Susanne und Katharina König (2016): Kommunikative Gattungen in der Interaktion: Kulturelle und grammatische Praktiken im Gebrauch. In: Deppermann, Arnulf, Helmuth Feilke und Angelika Linke (Hrsg.), Sprachliche und kommunikative Praktiken, S. 177-204. Berlin: de Gruyter.

Günthner, Susanne und Hubert Knoblauch (1994): ,Forms are the food of faith‘. Gattungen als Muster kommunikativen Handelns. In: Kölner Zeitschrift für Soziologie und Sozialpsychologie, 4:693-723.

Haddington, Pentti et al. (Hrsg.) (2014): Multiactivity in social interaction: Beyond multitasking. Amsterdam: Benjamins.

Heinemann, Trine und Jakob Steensig (2017): Three imperative action formats in Danish talk-ininteraction: The case of imperative + modal particles bare and lige. In: Sorjonen, Marja- 
Leena, Liisa Raevaara und Elizabeth Couper-Kuhlen (Hrsg.), Imperative turns at talk. The design of directives in action, S. 139-173. Amsterdam: Benjamins.

Heritage, John (2010): Conversation Analysis: Practices and Methods. In: Silverman, David (Hrsg.), Qualitative Sociology, S. 208-230. London: Sage, 3. Aufl.

Imo, Wolfgang (2007): Construction-Grammar und Gesprochene-Sprache Forschung. Tübingen: Niemeyer.

Imo, Wolfgang (2011): Die Grenzen von Konstruktionen: Versuch einer granularen Neubestimmung des Konstruktionsbegriffs der Construction Grammar. In: Engelberg, Stefan, Anke Holler und Kristel Proost (Hrsg.), Sprachliches Wissen zwischen Lexikon und Grammatik. (Jahrbuch 2010 des IDS), S. 113-148. Berlin: de Gruyter.

Imo, Wolfgang (2015): Interactional Construction Grammar. In: Linguistic Vanguard, 1(1):69-77.

Kay, Paul und Charles J. Fillmore (1999): Grammatical constructions and linguistic generalizations: The What's X doing Y? construction. In: Language, 75(1):1-33.

Langacker, Ronald W. (1987): Foundations of cognitive grammar. Vol. 1: Theoretical prerequisites. Stanford, CA: Stanford University Press.

Langacker, Ronald W. (2008): Cognitive Grammar. Oxford: OUP.

Lindwall, Oskar, Gustav Lymer und Christian Greiffenhagen (2015): The sequential analysis of instruction. In: Markee, Numa (Hrsg.), The handbook of classroom discourse and interaction, S. 142-157. Hoboken, NJ: Wiley.

Linke, Angelika (i. d. B.): Musterwandel als Indikator für soziokulturellen Wandel. Ein Abriss zur Veränderung von Grussformeln vom 17. zum 21. Jahrhundert. In: Weidner, Beate et al. (Hrsg.), Verfestigungen in der Interaktion. Konstruktionen, sequenzielle Muster, kommunikative Gattungen. Berlin: de Gruyter.

Luckmann, Thomas (1986): Grundformen der gesellschaftlichen Vermittlung des Wissens: Kommunikative Gattungen. In: Zeitschrift für Soziologie, 27:191-211.

Mondada, Lorenza (2011): The situated organisation of directives in French: Imperatives and action coordination in video games. In: Nottingham French Studies, 50(2):19-50.

Mondada, Lorenza (2013): Coordinating mobile action in real time: the timely organization of directives in video games. In: Haddington, Pentti, Lorenza Mondada und Maurice Nevile (Hrsg.), Interaction and mobility, S. 300-341. Berlin: de Gruyter.

Mondada, Lorenza (2017): Imperatives in action. In: Couper-Kuhlen, Elizabeth, Liisa Raevaara und Marja-Leena Sorjonen (Hrsg.), Imperatives in interaction, S. 65-101. Amsterdam: John Benjamins.

Mondada, Lorenza (2018): Driving instruction at high speed on a race circuit: Issues in action formation and sequence organization. In: International Journal of Applied Linguistics, 28(2):304-325.

Östman, Jan-Ola (2015): From Construction Grammar to Construction Discourse... and back. In: Bücker, Jörg, Susanne Günthner und Wolfgang Imo (Hrsg.), Konstruktionsgrammatik V: Konstruktionen im Spannungsfeld von sequenziellen Mustern, kommunikativen Gattungen und Textsorten, S. 15-44. Tübingen: Stauffenburg Verlag.

Proske, Nadine (2014): ${ }^{\circ} \mathrm{h}$ ach KOMM; hör AUF mit dem kIElnkram. Die Partikel komm zwischen Interjektion und Diskursmarker. In: Gesprächsforschung - Online-Zeitschrift zur verbalen Interaktion, 15:121-160.

Proske, Nadine (2017): Zur Funktion und Klassifikation gesprächsorganisatorischer Imperative. In: Blühdorn, Hardarik et al. (Hrsg.), Diskursmarker im Deutschen. Reflexionen und Analysen, S. 73-101. Göttingen: Verlag für Gesprächsforschung. 
Raffelsiefen, Renate (2002): Imperatives: the relation between meaning and form. In: Restle, David und Dietmar Zaefferer (Hrsg.), Sounds and systems: studies in structure and change, S. 319-345. Berlin: Mouton de Gruyter.

Rauniomaa, Mirka (2017): Assigning roles and responsibilities. In: Sorjonen, Marja-Leena, Liisa Raevaara und Elizabeth Couper-Kuhlen (Hrsg.), Imperative Turns at Talk: The design of directives in action, S. 325-355. Amsterdam: John Benjamins.

Raymond, Geoffrey (2003): Grammar and social organization: Yes/no interrogatives and the structure of responding. In: American Sociological Review, 68:939-967.

Reckwitz, Andreas (2003): Grundelemente einer Theorie sozialer Praktiken. In: Zeitschrift für Soziologie, 32:182-201.

Rossi, Giovanni (2012): Bilateral and unilateral requests: The use of imperatives and $\mathrm{mi} x$ ? interrogatives in Italian. In: Discourse Processes, 49:426-458.

Rossi, Giovanni (2015): The request system in Italian interaction. PhD Thesis, Radboud University, Nijmegen.

Schatzki, Theodore R., Karin Knorr Certina und Eike von Savigny (Hrsg.) (2001): The practice turn in contemporary theory. London: Routledge.

Schegloff, Emanuel A. (1984): On some questions and ambiguities in conversation. In: Atkinson, John Maxwell und John Heritage (Hrsg.), Structures of social action. Studies in conversation analysis, S. 28-52. Cambridge: CUP.

Schegloff, Emanuel A. (1996): Turn organization. One intersection of grammar and interaction. In: Ochs, Elinor, Emanuel A. Schegloff und Sandra A. Thompson (Hrsg.), Interaction and grammar, S. 52-133. Cambridge: CUP.

Schegloff, Emanuel A. (1997): Practices and actions. Boundary cases of other-initiated repair. In: Discourse Processes, 23(3):499-545.

Schmidt, Thomas (2018): Gesprächskorpora. In: Kupietz, Marc und Thomas Schmidt (Hrsg.), Korpuslinguistik, S. 209-230. Berlin: de Gruyter.

Selting, Margret (2016): Praktiken des Sprechens und Interagierens im Gespräch aus der Sicht von Konversationsanalyse und Interaktionaler Linguistik. In: Deppermann, Arnulf, Helmuth Feilke und Angelika Linke (Hrsg.), Sprachliche und kommunikative Praktiken, S. 27-56. Berlin: de Gruyter.

Sorjonen, Merja-Leena (2017): Imperatives and responsiveness in Finnish conversation. In: Sorjonen, Marja-Leena, Liisa Raevaara und Elizabeth Couper-Kuhlen (Hrsg.), Imperative turns at talk. The design of directives in action, S. 241-270. Amsterdam: Benjamins.

Stevanovic, Melisa (2017): Managing compliance in violin instruction. The case of the Finnish clitic particles -pA and -pAs in imperatives and hortatives. In: Sorjonen, Marja-Leena, Liisa Raevaara und Elizabeth Couper-Kuhlen (Hrsg.), Imperative turns at talk. The design of directives in action, S. 357-380. Amsterdam: Benjamins.

Stevanovic, Melisa und Anssi Peräkylä (2012): Deontic authority in interaction: the right to announce, propose, and decide. In: Research on Language and Social Interaction, 45(4):297-321.

Stivers, Tanya und Federico Rossano (2010): Mobilizing Response. In: Research on Language and Social Interaction, 43(1):3-31.

Stukenbrock, Anja (2015): Deixis in der face-to-face Interaktion. Berlin: de Gruyter.

Stukenbrock, Anja (i. d. B.): Mit Blick auf die Geste - multimodale Verfestigungen in der Interak-tion. In: Weidner, Beate et al. (Hrsg.), Verfestigungen in der Interaktion. Konstruktionen, sequenzielle Muster, kommunikative Gattungen. Berlin: de Gruyter. 
Thompson, Sandra A., Barbara A. Fox und Elizabeth Couper-Kuhlen (2015): Grammar in Everyday Talk: Building Responsive Actions. Cambridge: CUP.

Vine, Bernadette (2009): Directives at work: Exploring the contextual complexity of workplace directives. In: Journal of Pragmatics, 41(7):1395-1405.

Zifonun, Gisela, Ludger Hoffmann und Bruno Strecker (1997): Grammatik der deutschen Sprache. Berlin: de Gruyter.

Zinken, Jörg (2016): Requesting responsibility. The morality of grammar in Polish and English family interaction. Oxford: OUP.

Zinken, Jörg und Arnulf Deppermann (2017): A cline of visible commitment in the situated design of imperative turns. Evidence from German and Polish. In: Sorjonen, Marja-Leena, Liisa Raevaara und Elizabeth Couper-Kuhlen (Hrsg.), Imperative turns at talk. The design of directives in action, S. 27-63. Amsterdam: Benjamins.

Zinken, Jörg und Eva Ogiermann (2013): Responsibility and action. Invariants and diversity in object requests in Polish and British English interaction. In: Research on Language and Social Interaction, 46(3):256-276. 
